\title{
Communication Using a Large-Scale Array of Ubiquitous Antennas: A Geometry Approach
}

\author{
Kaibin Huang, Jiayi Chen and Vincent K. N. Lau
}

\begin{abstract}
The recent trends of densification and centralized signal processing in radio access networks suggest that future networks may comprise ubiquitous antennas coordinated to form a network-wide gigantic array, referred to as the ubiquitous array (UA). In this paper, the UA communication techniques are designed and analyzed based on a geometric model. Specifically, the UA is modeled as a continuous circular/spherical array enclosing target users and free-space propagation is assumed. First, consider the estimation of multiuser UA channels induced by user locations. Given single pilot symbols, a novel channel estimation scheme is proposed that decomposes training signals into Fourier/Laplace series and thereby translates multiuser channel estimation into peak detection of a derive function of location. The process is shown to suppress noise. Moreover, it is proved that estimation error due to interference diminishes with the increasing minimum user-separation distance following the power law, where the exponent is $1 / 3$ and 1 for the circular and spherical UA, respectively. If orthogonal pilot sequences are used, channel estimation is found to be perfect. Next, consider channel-conjugate data transmission that maximizes received signal power. The power of interference between two users is shown to decay with the increasing user-separation distance sub-linearly and super-linearly for the circular and spherical UA, respectively. Furthermore, a novel multiuser precoding design is proposed by exciting different phase modes of the UA and controlling the mode weight factors to null interference. The number of available degrees of freedom for interference nulling using the UA is proved to be proportional to the minimum user-separation distance.
\end{abstract}

\section{INTRODUCTION}

The explosive growth of mobile traffic is driving the rapid network densification to provide high-speed wireless access to coverage regions. To rein in the escalating network cost and interference, wireless access networks are evolving towards having an architecture with centralized signal processing and minimum on-site hardware comprising merely antennas and RF units, called either cloud radio access networks or base-station virtualization [1]. The network evolution as well as the advancements of other technologies

K. Huang is with the Dept. of Electrical and Electronic Engineering at The University of Hong Kong, Hong Kong (Email: huangkb@eee.hku.hk). J. Chen is with the College of Information Engineering, Shenzhen University, China (Email: i.c.jiayi@ieee.org). V. K. N. Lau is with the Dept. of Electrical and Computer Engineering at The Hong Kong University of Science and Technology, Hong Kong (Email: eeknlau@ece.ust.hk). Updated on September 2, 2018. 
such as small cells [2] and massive MIMO [3] will lead to future networks where antennas are ubiquitous and have network-wide coordination to form a gigantic array, which is called the ubiquitous array (UA) and forms the theme for the paper.

\section{A. Prior Work and New Challenges}

The UA system is equivalent to a distributed antennas system (DAS) with dense antennas and without cells. DASs refers to cellular systems where in each cell, antennas are distributed over the cell region and connected to a central processing unit [4]. This technology was initially developed to reduce transmission power and improve network coverage by either simulcast over all distributed antennas or serve each user using the nearest antennas [5], [6]. Recent research on DASs focuses on increasing the sum rate based on multiuser MIMO transmission using the distributed antennas as a virtual array, addressing issues such as inter-cell interference distribution [7], resource allocation [8], capacity analysis [9]-[11] and multi-cell coordination [12]. Each MIMO channel in such systems has coefficients corresponding to heterogeneous path loss depending on antenna locations and cannot be modeled as i.i.d. random variable as for the case of co-located antennas (see e.g., [13]). This complicates the distributions of signals and interference [7] and provides extra degrees of freedom, namely antenna locations, for sum-rate optimization [14], [15]. Essentially, this work is an attempt to quantify the advantages of extremely dense distributed antennas for channel estimation and data transmission.

Given the proximity between the UA and users, the UA channel is typically over free space or at most contains sparse scatterers. Combining free space channels and ubiquitous antennas shifts the paradigm of MIMO communications in several aspects. First, a new approach is needed for analyzing the capacity of the UA channel. Rich scattering is commonly assumed in a conventional MIMO channel, allowing the channel to be modeled as a random matrix and its capacity analyzed using probability theory and linear algebra (see e.g., [13]). This approach, however, is unsuitable for the UA channel with free space propagation since the channel capacity depends on the array geometry and the user locations. Thus, analyzing the capacity of the UA channel should rely on an approach merging geometry, electromagnetic wave theory and information theory in the same vein as [16], [17]. Next, despite the massive number of elements in the UA, the UA channel over free space is determined only by a few parameters such as the user locations and this fact can be exploited to dramatically reduce the complexity of channel estimation. In contrast, given rich scattering, deploying more antennas leads to continuous growth of the number of degrees of freedom (DoF) in the channel, which makes channel estimation a key challenge in designing massive MIMO systems [18]. Last, the classic technique for multiuser beamforming for free space channels computes nulls by solving a linear system where the number of variables is equal to that of transmit antennas [19], and thus is inefficient for the UA system with a massive number of transmit antennas. This calls for the design of efficient transmission algorithms for the UA systems. 


\section{B. Contributions and Organization}

The paper represents the first attempt on designing the UA communication systems and focuses on the signal-processing aspect, namely channel estimation and data transmission. For tractability, the work considers a particular coverage region represented by a simple geometric model which comprises a continuous circular UA communicating with $U$ single-antenna users at fixed locations near the UA center. In the model, propagation is constrained to be within the horizontal plane. The results are subsequently extended to the case with propagation in the three-dimensional space and a continuous spherical UA. Channels are assumed to be free space, narrow band, and reciprocal. The elements of the UA and user antennas are all assumed to be omnidirectional. The layout of the above model is similar to that of some existing ones for single-cell DASs (see e.g., [21]) that, however, assumes discrete antennas and rich scattering. The continuity of the UA, modeling dense antennas, is a typical technique for avoiding consideration of antenna placement (see e.g., [16]). More important, it is instrumental for the new findings and the algorithmic designs as summarized in the sequel.

First, consider estimation of multiuser UA channels using only single pilot symbols. The channels are determined by user locations and thus called location induced channels (LI-channels). The channel responses are non-linear functions of the locations. This makes the conventional linear (mimimm-meansquare-error or zero-forcing) estimation unsuitable and the optimal maximum-a-posteriori estimation intractable since it requires solving a set of non-linear equations [22]. To address this issue, a novel lowcomplexity channel-estimation technique is proposed based on decomposing the receive multiuser circular training signal into a Fourier series for the circular UA or spherical harmonics for the spherical UA. This leads to a derived function of location, called the user-location profile. The proposed estimation method is to detect the locations of the peaks of the profile that yield estimated user locations. The estimation procedure is shown to suppress noise by averaging and incur estimation errors only due to interference between multi-channel etimation. The error is shown to decay with the minimum user-separation distance following the power law with the exponent $1 / 3$ and 1 for the circular and spherical UA, respectively. Therefore, without orthogonal pilot sequences, multiuser channel estimation in the UA system is enabled by sufficiently large user-separation distances instead of differentiation in multiuser angles of arrival as in the conventional MIMO systems (see e.g., [23]). In addition, applying the method to the scenario where users deploy orthogonal pilot sequences leads to almost-perfect channel estimation.

Next, consider channel conjugate data transmission using the UA. The signal-to-interference-and-noise ratios (SINRs) are derived in closed-form. In particular, the power of interference between any two users is shown be proportional to their separation distance (in wavelength) raised to the power of $2 / 3$ and 2, corresponding to the circular and spherical UA, respectively. Therefore, even given single-user transmission, interference can be suppressed by increasing users' separation distances. Moreover, the path lose is shown to be inversely proportional to the propagation distance or fixed regardless of the distance for the circular and spherical UAs, respectively. In contrast, the loss for a conventional array 
with collocated elements is inversely proportional to the squared distance.

Last, a novel low-complexity precoding design is proposed for multiuser transmission using the UA. Specifically, the precoders are designed in the form of Fourier series for the circular UA and spherical harmonics for the spherical UA. Their coefficients are controlled as derived to excite different phase modes of the circular array so as to null multiuser interference. For this sub-optimal design, the number of DoF available for interference nulling is shown to be proportional to the minimum user separation distance or its square for the circular and spherical UAs, respectively.

The reminder of the paper is organized as follows. The UA system model is described in Section II. Algorithms for channel estimation and data transmission for the circular-UA system are presented in Section III and IV, respectively. The results are extended to the spherical-UA system in Section V. Simulation results are provided in Section VI follows by concluding remarks in Section VII. In Appendix A, Bessel functions and spherical harmonics are defined and their properties discussed. Last, Appendix B contains the proofs for lemmas.

\section{SySTEM MODEL}

As illustrated in Fig. 1, the UA is modeled as either a circular array or a spherical array, denoted as $\mathrm{O}$ and $\mathrm{O}^{2}$, respectively, having the same radius denoted as $r_{0}$. The dense UA is assumed to be continuous for tractable analysis but this assumption is relaxed in simulation. The communication system comprises the UA centered at the origin and a set of $U$ single-antenna users enclosed by the UA, represented by their fixed locations $X_{1}, X_{2}, \cdots, X_{U}$ in the horizontal plane. A user, $X_{u}$, and a particular element of the UA, $A$, are represented by their spherical coordinates $\left(r_{u}, \varphi_{u}, \frac{\pi}{2}\right)$ and $\left(r_{0}, \varphi, \theta\right)$, respectively, where $\left(\varphi_{u}, \varphi\right)$ are azimuth angles and $\left(\frac{\pi}{2}, \theta\right)$ are polar angles with $\theta=\frac{\pi}{2}$ for the case of circular UA. In addition, there are no scatterers.

Assumption 1. Users are located near the center of the UA such that $r_{u} / r_{0} \ll 1$ for $u=1, \cdots, U$.

The assumption allows tractable analysis as it simplifies the expression for the propagation distances. Specifically, given a user $X_{u}$ and a UA antenna $A$, the separation distance and angle are denoted as $\left|X_{u}-A\right|$ and $\psi_{u}(A)$, respectively, with

$$
\begin{aligned}
\left|X_{u}-A\right| & =\sqrt{r_{0}^{2}+r_{u}^{2}-2 r_{0} r_{u} \cos \psi_{u}(A)} \\
& =r_{0}-r_{u} \cos \psi_{u}(A)+o
\end{aligned}
$$

where $o$ represents $O\left(\max _{u} r_{u} / r_{0}\right)$. Note that the above model can be extended to include a set of scatterers at given locations, which reflect communication signals between the UA and users in the absence of lines of sight. Remarks on the extensions of results to scattering channels are provided in the sequel.

The wave transmitted by an antenna is assumed to propagate as a plane wave in the three-dimensional free space. All antennas are assumed to be omni-directional. Let $h_{u}(A)$ represents the response of the 


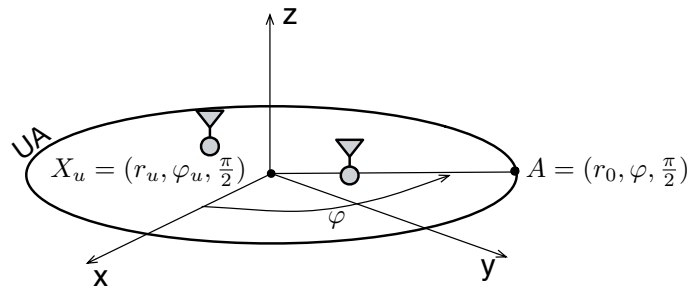

(a) Circular-UA System

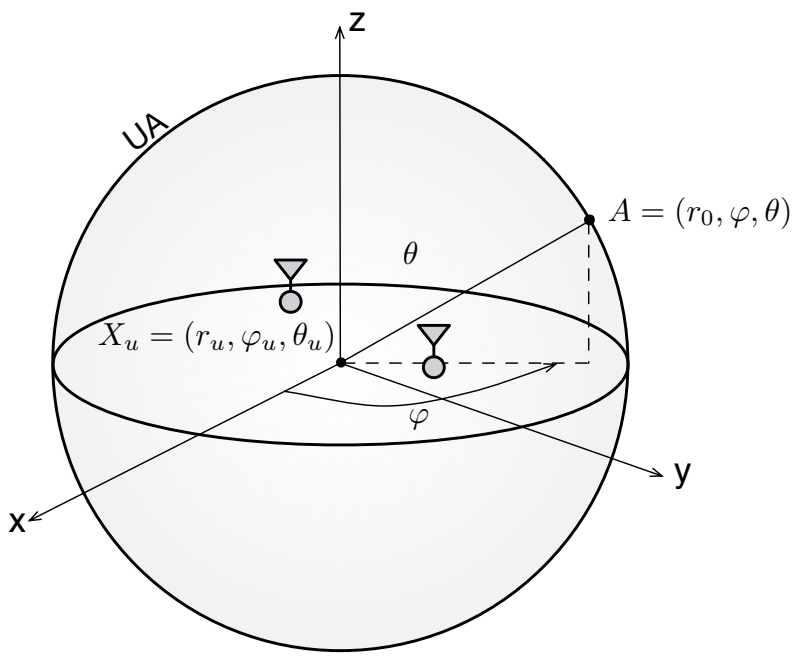

(b) Spherical-UA System

Fig. 1. A geometric model of a communication system using a continuous circular/spherical UA. channel between $X_{u}$ and $A$. As a result,

$$
h_{u}(A)=\frac{1}{\sqrt{4 \pi}\left|X_{u}-A\right|} e^{-j \frac{2 \pi}{\lambda}\left|X_{u}-A\right|}, \quad A \in \mathbb{O} \text { or } \mathbb{O}^{2}
$$

where $\lambda$ denotes the carrier wavelength. Based on (1),

$$
h_{u}(A)=\frac{1}{\sqrt{4 \pi} r_{0}} e^{-j \frac{2 \pi}{\lambda} r_{0}+j \frac{2 \pi}{\lambda} r_{u} \psi_{u}(A)}+\frac{o}{r_{0}}, \quad A \in \mathbb{O} \text { or } \mathbb{O}^{2} .
$$

Channel estimation at the UA is assisted by pilot signals transmitted by users. Time is divided into slots with unit symbol durations. Since the effective aperture for a omni-directional antenna is $\lambda^{2} / 4 \pi$ [24], the total pilot signal received at antenna $A$ in an arbitrary slot, denoted as $q(A)$, is given as

$$
q(A)=\frac{\lambda}{\sqrt{4 \pi}} \sum_{u=1}^{U} h_{u}(A) s_{u}+z(A), \quad A \in \mathbb{O} \text { or } \mathbb{O}^{2}
$$

where $s_{u}$ is a pilot symbol transmitted by user $u$ and the noise $z(A)$ is a spatial sample of the additive white Gaussian noise $\mathcal{C N}\left(0, \sigma^{2}\right)$ process at location $A$. The noise processes are assumed to be spatially white as follows.

Assumption 2. The noise processes $z(X)$ and $z(Y)$ at two locations $X$ and $Y$ are independent if $X \neq Y$.

Substituting (2) into (3) gives

$$
q(A)=\frac{\lambda e^{-j \frac{2 \pi}{\lambda} r_{0}}}{4 \pi r_{0}} \sum_{u=1}^{U} e^{j \frac{2 \pi}{\lambda} r_{u} \psi_{u}(A)} s_{u}+z(A)+\frac{o}{r_{0}}, \quad A \in \mathbb{O} \text { or } \mathbb{O}^{2} .
$$

Next, consider downlink data transmission. The data symbol intended for user $u$ is denoted as $x_{u}$ and assumed to be distributed as a $\mathcal{C N}(0,1)$ random variable. The symbol is precoded by a continuous precoder represented by $f_{u}: \mathbb{O}$ or $\mathbb{O}^{2} \rightarrow \mathbb{C}$. Let $P_{\mathrm{t}}$ denote the transmission power per user. Then the incident field at location $u$ in an arbitrary slot, denoted as $g\left(X_{u}\right)$, can be written as

$$
g\left(X_{u}\right)=\sqrt{\frac{P_{t}}{\partial \mathcal{A}}} \int_{\mathcal{A}} h_{u}(A) \sum_{k=1}^{U} f_{k}(A) d A x_{k}, \quad \mathcal{A}=\mathbb{O} \text { or } \mathbb{O}^{2}
$$


with $h_{u}(A)$ being the channel response in (2) and $\partial \mathcal{A}$ represents the circumference of a circular UA $(\mathcal{A}=\mathbb{O})$ or the surface area of a spherical UA $\left(\mathcal{A}=\mathbb{O}^{2}\right)$. It follows that the corresponding received signal is

$$
y_{u}=\frac{\lambda}{\sqrt{4 \pi}} g\left(X_{u}\right)+z_{u}, \quad u=1,2, \cdots, U
$$

where $\left\{z_{u}\right\}$ are i.i.d. $\mathcal{C N}\left(0, \sigma^{2}\right)$ random variables representing channel noise.

\section{Communication Using the Circular UA: Channel Estimation}

Estimation of the LI-channels is to infer the user locations from the training signal, namely

$$
\{q(A) \mid A \in \mathbb{O}\} \Longrightarrow\left\{X_{u}\right\}
$$

with $\{q(A)\}$ given in (4). As mentioned, the linear or MAP estimation techniques are intractable since $q(A)$ is a nonlinear function of $\left\{X_{u}\right\}$. To address this issue, a simple estimation scheme is proposed in the following sub-sections, which reduces channel estimation to the detection of the peaks of a given function.

\section{A. LI-Channel Estimation with Single Pilot Symbols}

Consider the scenario where users simultaneously transmit single pilot symbols $\left\{s_{u}\right\}$ to facilitate channel estimation at the UA. Without loss of generality, assume that the pilot symbols are all ones: $s_{u}=1 \forall u$. Let the training signal $q(A)$ in (3) be re-denoted as $q(\varphi)$ since $A=\left(r_{0}, \varphi, \frac{\pi}{2}\right)$.

The LI-channel estimation scheme is designed as follows. First, the proposed scheme is based on decomposing the received training signal $\{q(\varphi)\}$ into a Fourier series:

$$
q(\varphi)=\sum_{k=-\infty}^{\infty} Q_{k} e^{-j k \varphi}, \quad \varphi \in[0,2 \pi]
$$

where the Fourier coefficients $\left\{Q_{k}\right\}$ are defined as

$$
Q_{k}=\frac{1}{2 \pi} \int_{0}^{2 \pi} q(\varphi) d \varphi
$$

The Fourier coefficients contain all information on the signal and thus can replace it in channel estimation. To facilitate the algorithmic design, the structure of the coefficients is characterized as follows. To this end, a few notations are introduced. Let $\mathbf{Q}$ represent the infinite sequence: $\cdots, Q_{-1}, Q_{0}, Q_{1}, \cdots$. The product between two sequences, $\mathbf{V}_{1}$ and $\mathbf{V}_{2}$, is denoted and defined as $\mathbf{V}_{1} \circ \mathbf{V}_{2}=\sum_{n=-\infty}^{\infty}\left[\mathbf{V}_{1}\right]_{n}^{*}\left[\mathbf{V}_{2}\right]_{n}$ where $[\cdot]_{n}$ yields the element with index $n$. Moreover, $J_{n}: \mathbb{R} \rightarrow \mathbb{R}$ represents Bessel function with an integer index $n$ as defined and discussed in Appendix A.

Lemma 1 (Training signal decomposition). The sequence $\mathbf{Q}$ can be decomposed as

$$
\mathbf{Q}=\frac{\lambda}{4 \pi r_{0}} e^{-j \frac{2 \pi}{\lambda} r_{0}} \sum_{u=1}^{U} \mathbf{V}\left(X_{u}\right)+\frac{o}{r_{0}}, \quad \text { a.s. }
$$


where $\mathbf{V}\left(X_{u}\right)=\cdots V_{-1}\left(X_{u}\right), V_{0}\left(X_{u}\right), V_{1}\left(X_{u}\right) \cdots$ with the function $V_{n}(Y)$ defined for a given location $Y=\left(r_{Y}, \varphi_{Y}, \frac{\pi}{2}\right)$ as

$$
V_{n}(Y)=j^{n} e^{j n \varphi_{Y}} J_{n}\left(\frac{2 \pi}{\lambda} r_{Y}\right)
$$

The proof is provided in Appendix B-A.

Remark 1. The Fourier coefficients $\left\{Q_{k}\right\}$ of the received training signal are noiseless. The noise suppression is the combined result of the noise spatial whiteness in Assumption 2 and the integral operation in (8).

Next, based on the decomposition of $\mathbf{Q}$ in Lemma 1, the key component of the proposed scheme for LI-channel estimation is a function $\Phi: \mathbb{R}^{2} \rightarrow \mathbb{R}_{+}$defined as

$$
\Phi(Y)=\frac{4 \pi r_{0}}{\lambda}|\mathbf{V}(Y) \circ \mathbf{Q}|
$$

and called the channel observation profile . To derive a closed-form expression for $\Phi(Y)$, a useful property for $\mathbf{V}$ directly follows from the Addition Theorem in Property (B2) of Bessel functions in Appendix A as shown below.

Lemma 2. Given two locations $X, Y \in \mathbb{R}^{2}$, the product between the sequences $\mathbf{V}(X)$ and $\mathbf{V}(Y)$ satisfies

$$
|\mathbf{V}(X) \circ \mathbf{V}(Y)|=J_{0}\left(\frac{2 \pi}{\lambda}|X-Y|\right) .
$$

Combining Lemmas 1 and 2 and the definition of $\Phi(Y)$ in (11) gives the following theorem.

Theorem 1 (Channel observation). The channel observation profile corresponding to the circular UA is noiseless and given as

$$
\Phi(Y)=\left|\sum_{u=1}^{U} J_{0}\left(\frac{2 \pi}{\lambda}\left|X_{u}-Y\right|\right)\right|+o, \quad \text { a.s. }
$$

An example of $\Phi(Y)$ is illustrated in Fig. 2.

LI-channel estimation scheme: One can observe from Theorem 1 that the $U$ Bessel functions in $\Phi(Y)$ have their peaks at corresponding user locations since $J_{0}(x)$ is maximized at $x=0$. Motivated by this fact, the proposed scheme for estimating the user locations is to detect the peaks in the channel observation profile $\Phi(Y)$. Since the profile is noiseless, the only source for estimation errors is the coupling (interference) between the Bessel functions in $\Phi(Y)$ due to finite separation distances between user locations.

Remark 2 (Channel estimation error). Channel estimation using the proposed scheme is close to perfect for a single-user system since the corresponding channel observation profile $\Phi(Y) \approx J_{0}\left(\frac{2 \pi}{\lambda}\left|X_{1}-Y\right|\right)$ that is maximized at $Y=X_{1}$ with $\Phi\left(X_{1}\right) \approx 1$. Next, consider the estimation of multiuser LI-channels. The accuracy for estimating the channel corresponding to user $X_{u}$ can be evaluated by the difference 


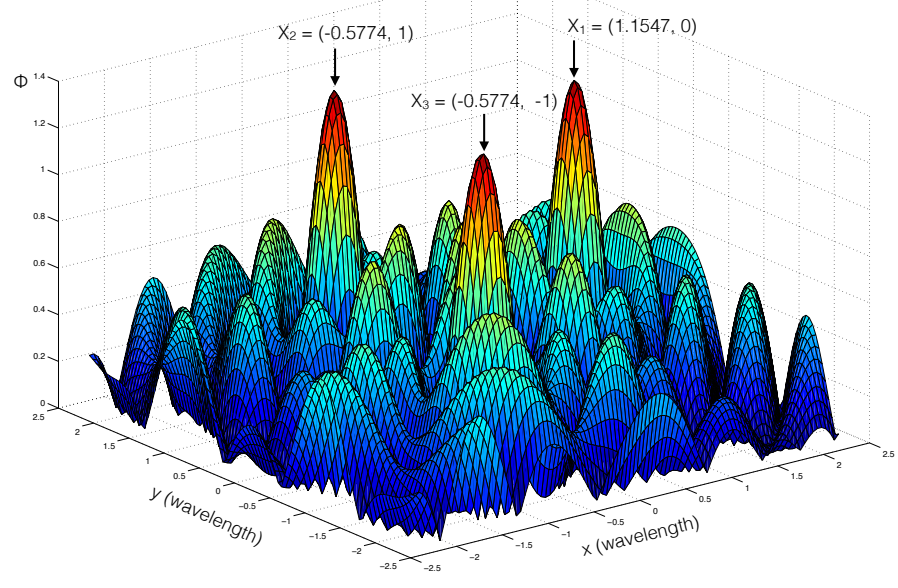

Fig. 2. An example of the channel observation profile corresponding to three users with equal separation distances of 2 wavelengths where the peaks are identified by their Cartesian coordinates and the ripples arise from the tails of superimposed Bessel functions (see Theorem 1).

between $\Phi\left(X_{u}\right)$ and the value of 1 for its single-user counterpart. This measures the interference due to the presence of multiuser channels and can be obtained from Theorem 1 as follows:

$$
\left|\Phi\left(X_{u}\right)-1\right| \leq\left|\sum_{k \neq u} J_{0}\left(\frac{2 \pi}{\lambda}\left|X_{k}-X_{u}\right|\right)\right|+o, \quad \text { a.s. }
$$

Based on Property (B4) of Bessel functions in Appendix A,

$$
\begin{aligned}
\left|\Phi\left(X_{u}\right)-1\right| & \leq \frac{1}{\nu} \sum_{k \neq u}\left(\frac{2 \pi}{\lambda}\left|X_{k}-X_{u}\right|\right)^{-\frac{1}{3}}+o, \quad \text { a.s. } \\
& \leq \frac{U-1}{\nu}\left(\frac{2 \pi}{\lambda} \min _{u \neq k}\left|X_{k}-X_{u}\right|\right)^{-\frac{1}{3}}+o, \quad \text { a.s. }
\end{aligned}
$$

The result shows that the interference magnitude diminishes with the increasing minimum user-separation distance approximately following a sub-linear function. Given a pair of users, setting the upper bound in (13) to a small value e.g., 0.1, a rule of thumb for the user-separation distance sufficiently large for accurate multiuser channel estimation can be computed as $77 \lambda$, which is $23 \mathrm{~m}$ for a carrier of $1 \mathrm{Gz}$ and $2.3 \mathrm{~m}$ for $10 \mathrm{Gz}$.

Remark 3 (Effect of wavelength on channel estimation). One can infer from (13) that with user locations fixed, the accuracy of channel estimation can be improved by increasing the carrier wavelength $\lambda$. However, this leads to a denser UA in practice since its elements are required to be separated by no more than $\lambda / 2$.

Remark 4 (Scattering Channels). Assuming the reflection at scatterers are isotropic, the channels between the UA and users are determined not only by the scatterers' locations but also by the channel coefficients that combine the gains of the channels between scatterers and users and reflection attenuation at scatterers. Estimation of the channels can follow a two-phase procedure. First, the scatter locations can be estimated 
following the same scheme as discussed earlier for estimating user locations. Next, resolving the scatter locations allows the estimation of the training signals reflected by individual scatterers. However, further estimating the individual channel coefficient between each pair of scatterer and user requires the use of pilot sequences and exploiting their orthogonality. In other words, unlike that of free-space channels, channel estimation using single pilot symbols is infeasible for scattering channels.

\section{B. LI-Channel Estimation with Pilot Sequences}

In this section, the results in the preceding section for single pilot symbols are extended to the case with pilot sequences. Let the pilot sequence for the $u$-th user be denoted as $\mathbf{s}_{u}=\left[s_{u}(1), \cdots, s_{u}(L)\right]^{T}$ with length $L$. In the channel training phase, the UA receives sequentially $L$ infinite sequences over $L$ symbol durations, denotes as $\mathbf{Q}(1), \mathbf{Q}(2), \cdots, \mathbf{Q}(L)$, where $\mathbf{Q}(\ell)$ is modified from its single-symbol counterpart in Lemma 1 as

$$
\mathbf{Q}(\ell)=\frac{\lambda e^{-j \frac{2 \pi}{\lambda} r_{0}}}{4 \pi r_{0}} \sum_{u=1}^{U} s_{u}(\ell) \mathbf{V}\left(X_{u}\right)+\frac{o}{r_{0}}, \quad \text { a.s. }
$$

To estimate the user location $X_{u},\{\mathbf{Q}(\ell)\}$ are coherently combined using $\mathbf{s}_{u}$ as $\sum_{\ell=1}^{L} s_{u}^{*}(\ell) \mathbf{Q}(\ell)$. The result, denoted as $\mathbf{Q}_{u}$, follows from (14) as

$$
\mathbf{Q}_{u}=\frac{\lambda e^{-j \frac{2 \pi}{\lambda} r_{0}}}{4 \pi r_{0}} \sum_{k=1}^{U} \mathbf{s}_{u}^{\dagger} \mathbf{s}_{k} \mathbf{V}\left(X_{k}\right)+\frac{o}{r_{0}}, \quad \text { a.s. }
$$

A corresponding channel observation profile $\Phi_{u}(Y)$ can be defined similarly as in (11):

$$
\Phi_{u}(Y)=\frac{4 \pi r_{0}}{\lambda}\left|\mathbf{V}(Y) \circ \mathbf{Q}_{u}\right|
$$

The profile $\Phi_{u}(Y)$ is decomposed into the desired and interference terms as follows:

$$
\Phi_{u}(Y)=\left|J_{0}\left(\frac{2 \pi}{\lambda}\left|X_{u}-Y\right|\right)+\sum_{k \neq u} \mathbf{s}_{u}^{\dagger} \mathbf{s}_{k} J_{0}\left(\frac{2 \pi}{\lambda}\left|X_{k}-Y\right|\right)\right|^{2}+o
$$

Then the deviation of $\Phi_{u}(Y)$ from its single-user counterpart can been bounded as:

$$
\left|\Phi_{u}(Y)-J_{0}^{2}\left(\frac{2 \pi}{\lambda}\left|X_{u}-Y\right|\right)\right| \leqslant \sum_{k \neq u}\left|\mathbf{s}_{u}^{\dagger} \mathbf{s}_{k}\right| J_{0}\left(\frac{2 \pi}{\lambda}\left|X_{k}-Y\right|\right)+o, \quad \forall u .
$$

This leads to the following main result of this section.

Theorem 2 (Effect of pilot sequences). Given $L \geq U$ and orthogonal pilot sequences, the LI-channel estimation using the circular UA is almost perfect since the channel observation profile is approximately equal to the single-user counterpart:

$$
\left|\Phi_{u}(Y)-J_{0}\left(\frac{2 \pi}{\lambda}\left|X_{u}-Y\right|\right)\right| \leqslant o, \quad u=1,2, \cdots, U .
$$

Comparing Theorems 1 and 2, the advantage of pilot sequences over single pilot symbols lies in their capability of decoupling the estimation of multiuser LI-channels, thereby providing close-to-perfect channel estimation. 


\section{Effect of Finite Elements in the UA}

Consider a discrete circular UA with $N$ antennas uniformly placed on the circle centered at the origin and with the radius $r_{0}$. The discrete UA can be interpreted as a quantized version of the continuous UA with the quantization error bounded by $\pi / N$. Based on this interpretation and assuming $N$ is large, the analysis for the continuous UA can be straightforwardly extended to the case of discrete UA by including the quantization error. As a result, for channel estimation with single pilot symbols, the channel observation profile for the discrete UA, denoted as $\hat{\Phi}(Y)$, can be written as

$$
\hat{\Phi}(Y)=\Phi(Y)+O\left(\frac{1}{N}\right) .
$$

where $\Phi(Y)$ for the continuous UA is given in Theorem 1. Moreover, the result in Theorem 2 for the case of pilot sequences can be modified by replacing $o$ with $o+O(1 / N)$. Similarly, for data transmission using

the discrete UA, the receive SINR at user $u$, denoted as $\widehat{\operatorname{SINR}}_{u}$, can be shown to be $\widehat{\operatorname{SINR}}_{u}=\operatorname{SINR}_{u}+$ $O(1 / N)$ with $\mathrm{SINR}_{u}$ corresponding to the continuous UA. The above results suggest that with respect to the continuous counterpart, the discrete UA causes additional fluctuation in the channel observation profile and receive SNRs, which can potentially degrades the performance of channel estimation and data transmission.

\section{Communication Using the Circular UA: Data Transmission}

In this section, two precoding techniques are designed for the UA, namely the channel conjugate and the multiuser phase mode (MU-PM) precoding. For simplicity, it is assumed that the UA has perfect knowledge of the LI-channels.

\section{A. Channel Conjugate Transmission}

For channel conjugate transmission, the precoder applies a phase shift to each antenna for compensating propagation delay to achieve coherent combining at the target user location, which is similar to beamforming using a phase array. Specifically, the precoder $f_{u}(\varphi)$ is given as

$$
f_{u}(\varphi)=\frac{h_{u}^{*}(\varphi)}{\left|h_{u}(\varphi)\right|}, \quad \varphi \in[0,2 \pi)
$$

where $h(\varphi)$ is given in (2). It follows that

$$
f_{u}(\varphi)=e^{j \frac{2 \pi}{\lambda}\left(r_{0}-r_{u} \psi_{u}(\varphi)\right)}, \quad \varphi \in[0,2 \pi)
$$

The normalization $\left|f_{u}(\varphi)\right|^{2}=1$ facilitates the UA implementation under the per-element power constraint e.g., using a phase array.

The channel conjugate precoder $\left\{f_{u}(\varphi)\right\}$ shapes the distribution of the field power density such transmission power is concentrated in a small region centered at the target location $X_{u}$. Characterizing the distribution is useful for analyzing the precoder performance. To this end, consider the transmission of an unmodulated wave using the UA after precoding using $\left\{f_{u}(\varphi)\right\}$ in (20). Conditioned on the precoder, 
let $g\left(X_{k} \mid X_{u}\right)$ denote the resultant field measured at the location $X_{k}$. With the propagation distance in (1), it can be obtained that

$$
g\left(X_{k} \mid X_{u}\right)=\sqrt{\frac{P_{\mathrm{t}}}{2 \pi r_{0}}} \times \frac{1}{\sqrt{4 \pi r_{0}^{2}}} \times \int_{0}^{2 \pi} e^{j \frac{2 \pi}{\lambda}\left(-r_{0}+r_{u} \cos \psi_{u}(\varphi)\right)} f_{k}(\varphi) r_{0} d \varphi+\frac{o}{\sqrt{r_{0}}} .
$$

At the right-hand side of (21), the three factors of the dominant term correspond to the density of transmission power uniformly distributed over the UA, the propagation loss and the wave superposition at $X_{u}$, respectively. Substituting the precoder in (20) into (21) yields

$$
g\left(X_{k} \mid X_{u}\right)=\sqrt{\frac{P_{\mathrm{t}}}{2 r_{0}}} \times \frac{1}{2 \pi} \int_{0}^{2 \pi} e^{j \frac{2 \pi}{\lambda}\left(r_{u} \cos \psi_{u}(\varphi)-r_{k} \cos \psi_{k}(\varphi)\right)} d \varphi+\frac{o}{\sqrt{r_{0}}} .
$$

The field power density at location $X_{k}$ can be represented by $p\left(X_{k} \mid X_{u}\right)=\left|g\left(X_{k} \mid X_{u}\right)\right|^{2}$. Using (22), a closed-form expression for $p\left(X_{k} \mid X_{u}\right)$ is obtained as shown in the following lemma proved in Appendix B-B.

Lemma 3 (Field power density distribution). Given the circular UA and channel conjugate precoding targeting user $X_{u}$, the field power density measured at the user location $X_{k}$ is given as

$$
p\left(X_{k} \mid X_{u}\right)=\frac{P_{\mathrm{t}}}{2 r_{0}} J_{0}^{2}\left(\frac{2 \pi}{\lambda}\left|X_{u}-X_{k}\right|\right)+\frac{o}{r_{0}} .
$$

The result shows that the field power density function $p\left(X_{k} \mid X_{u}\right)$ depends only on the distance $\left|X_{k}-X_{u}\right|$ and thus can be rewritten as $p(d)$ with $d>0$ denotes the the distance from the location targeted by the precoder. Then the distribution of the field power density can be characterized by the function $p(d) / p(0)=J_{0}\left(\frac{2 \pi d}{\lambda}\right)$ that is plotted in Fig. 3. It can be observed from the figure that the channel-conjugate precoder shapes the field distribution such that most power is concentrated within a circular region centered at the target location and having a radius of half wavelength. The tail of the distribution function is undesirable as it causes interference to nearby unintended receivers. However, the envelop of the tail decays with distance $d$, allowing interference suppression by spatial separation as further discussed in the sequel.

With the field distribution in (3), the performance of the channel-conjugate precoding is readily analyzed in terms of receive SINRs as follows. Let $P_{\mathrm{r}}$ and $P_{\mathrm{i}}$ denote the signal and interference powers at user $X_{u}$, respectively. Since the effective aperture of the receive omni-directional antenna is $\lambda / 4 \pi, P_{\mathrm{r}}$ and $P_{\mathrm{i}}$ are given as

$$
P_{\mathrm{r}}=\frac{\lambda}{4 \pi} p\left(X_{u} \mid X_{u}\right) \quad \text { and } \quad P_{\mathrm{i}}=\frac{\lambda}{4 \pi} \sum_{k \neq u} p\left(X_{u} \mid X_{k}\right) .
$$

The receive SINR for user $X_{u}$ can be written in terms $P_{\mathrm{r}}$ and $P_{\mathrm{i}}$ as

$$
\operatorname{SINR}_{u}=\frac{P_{\mathrm{r}}}{P_{\mathrm{i}}+\sigma^{2}}
$$

Substituting Lemma 3, (24) into (25) yields the following main result of this section. 


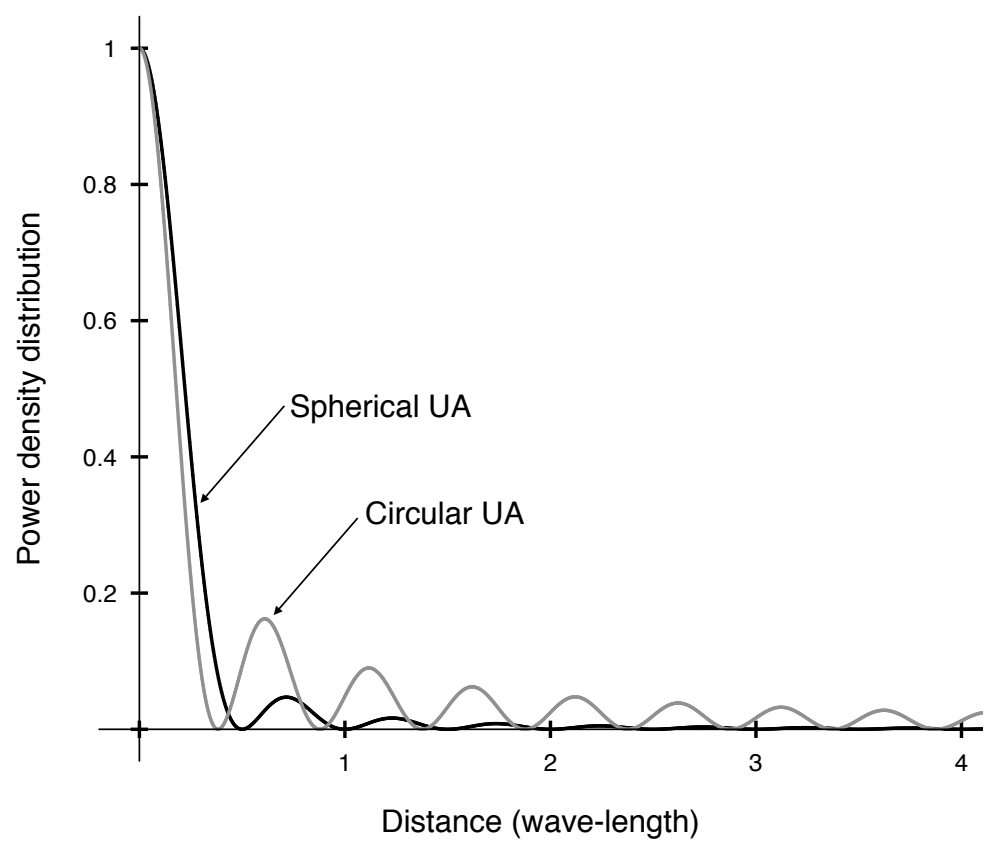

Fig. 3. Given channel-conjugate transmission, the distribution of field power density (normalized by its peak) as a function of distance from the peak location.

Theorem 3 (Receive SINRs). For channel conjugate transmission using the circular UA, the receive SINR for user $u$ is given as

$$
\operatorname{SINR}_{u}=\frac{1}{\sum_{k \neq u} J_{0}^{2}\left(\frac{2 \pi}{\lambda}\left|X_{u}-X_{k}\right|\right)+\frac{1}{\operatorname{SNR}}}+o, \quad u=1,2, \cdots, U
$$

where the receive $S N R$ is given as

$$
\mathrm{SNR}=\frac{P_{t} \lambda}{8 \pi \sigma^{2} r_{0}}+\frac{o}{r_{0}} .
$$

Remark 5 (High SNR). Applying the bound on the Bessel function in Property (B4) in Appendix A, for a high SNR, the signal-to-interference ratio (SIR) at user $u$ scales with the distance to the nearest interferer, namely $\min _{k \neq u}\left|X_{k}-X_{u}\right|$, and the wavelength $\lambda$ as

$$
\mathrm{SIR}_{u} \geqslant \frac{\nu^{2}}{U-1}\left(2 \pi \min _{k \neq u} \frac{\left|X_{k}-X_{u}\right|}{\lambda}\right)^{\frac{2}{3}}+o, \quad \forall u .
$$

Thus the receive SIRs increase with the increasing minimum user-separation distance (in wavelength) following a sub-linear function. Moreover, the result in (28) suggests that denser simultaneous users can be supported by reducing the wavelength without compromising the system throughput. Specifically, the user density can scale linearly with $1 / \lambda^{2}$.

Remark 6 (Free space vs. scattering channels). Free-space channels allow the UA to focus signal energy at intended users such that the signal power decays rapidly with the distance from the target location. As a result, given single-user (channel-conjugate) transmission, interference can be suppressed by increasing user spatial separation as shown in (28). However, this is infeasible in the scenario of scattering channels as scattering introduces additional cross coupling between multiuser signals. Suppressing the resultant 
interference cannot rely on increasing users' spatial separation and multiuser precoding has to be used for this purpose.

Remark 7 (High Mobility). Given fixed user locations, the sum rate is thus $\sum_{u} \log _{2}\left(1+\operatorname{SINR}_{u}\right)$ with $\mathrm{SINR}_{u}$ given in (26). For the scenario where users have high mobility, it is more appropriate to consider the ergodic sum rate given as

$$
\bar{R}=\mathrm{E}\left[\sum_{u} \log _{2}\left(1+\operatorname{SINR}_{u}\left(X_{1}, \cdots, X_{U}\right)\right)\right]
$$

where $\left\{X_{u}\right\}$ are random. The ergodic sum rate can be analyzed by combining the results in Theorem 3 and stochastic geometry [32].

Last, the received SNR given in (27) implies the following result.

Corollary 1 (Propagation loss). Given channel-conjugate transmission using the circular UA, the propagation loss is given as

$$
\frac{P_{\mathrm{r}}}{P_{\mathrm{t}}}=\frac{\lambda}{8 \pi r_{0}}+\frac{o}{r_{0}}
$$

In other words, transmission using the circular UA reduces the loss such that it is inversely proportional to the propagation distance instead of its square as in the case of a conventional array of collocated antennas.

\section{B. Multiuser Phase Mode Precoding}

In this section, MU-PM precoders are designed under the zero-forcing constraints to avoid multiuser interference. Exploiting the circular structure of the UA, the precoder for each user, say $f_{u}^{\prime}(\varphi)$ for user $u$, can be expressed in terms of the Fourier series representing the sequence of phase modes:

$$
f_{u}^{\prime}(\varphi)=f_{u}(\varphi) \sum_{m=-\infty}^{\infty} c_{u, m} e^{-j m \varphi}, \quad \varphi \in[0,2 \pi)
$$

where $f_{u}(\varphi)$ is the channel-conjugate precoder in (19) for compensating the channel phase shift and the summation is the said Fourier series. The precoder coefficients $\left\{c_{u, m}\right\}$ satisfy the power constraint: $\sum_{m=-\infty}^{\infty}\left|c_{u, m}\right|^{2} \leq 1$ for all $u$.

The precoder coefficients are designed to avoid the inter-user interference as follows. Without multiuser interference, it is unnecessary to analyze the field spatial distribution as in the case of channel conjugate transmission and instead the remainder of the section focuses on the precoder design and the analysis of the received signal power. To this end, the received signal at user $u$ is obtained by substituting the precoder in (31) into (5):

$$
y_{u}=\sqrt{\frac{\lambda P_{t}}{8 \pi r_{0}}} \sum_{k=1}^{U}\left(\frac{1}{2 \pi} \int_{0}^{2 \pi} \frac{h_{k}^{*}(\varphi) h_{u}(\varphi)}{\left|h_{k}(\varphi)\right|^{2}} \sum_{m=-\infty}^{\infty} c_{k, m} e^{-j m \varphi} d \varphi\right) x_{k}+z_{u} .
$$


The substitution of the channel response in (2) yields

$$
y_{u}=\sqrt{\frac{\lambda P_{t}}{8 \pi r_{0}}} c_{u, 0} x_{u}+\sqrt{\frac{\lambda P_{t}}{8 \pi r_{0}}} \sum_{k \neq u} \sum_{m=0}^{\infty} c_{k, m} \mathcal{J}_{u, k, m} x_{k}+z_{u}+\frac{o}{r_{0}}
$$

where the first term and the summation represent the signal and interference, respectively, and $\mathcal{J}_{u, k, m}$ is defined as

$$
\mathcal{J}_{u, k, m}=\frac{1}{2 \pi} \int_{0}^{2 \pi} e^{j \frac{2 \pi}{\lambda}\left(r_{u} \cos \left(\varphi_{u}-\varphi\right)-r_{k} \cos \left(\varphi_{k}-\varphi\right)\right)-j m \varphi} d \varphi
$$

A closed-form expression for $\mathcal{J}_{u, k, m}$ can be obtained as follows, following similar steps as in the proof for Lemma 3 with the details omitted for brevity.

Lemma 4. The coefficients $\left\{\mathcal{J}_{u, k, m}\right\}$ defined in (33) can be written as

$$
\left.\mathcal{J}_{u, k, m}=e^{j m \beta_{u, k}} J_{m}\left(\frac{2 \pi}{\lambda}\left|X_{u}-X_{k}\right|\right)\right)
$$

where $1 \leq u, k \leq U$ and $m$ is an integer.

The interference term in the signal in (32) can be nulled by enforcing the following zero-forcing constraints:

$$
\sum_{m=-\infty}^{\infty} c_{k, m} \mathcal{J}_{u, k, m}=0, \quad \forall k \neq u
$$

In practice, given a discrete UA and a constraint on computation complexity, it is infeasible to implement MU-PM precoders with an infinite number of phase modes and only a finite set of modes is considered in the design. Let the corresponding coefficient set be denoted as $\left\{c_{u, m} \mid 1 \leq u \leq U,-M \leq m \leq M\right\}$ with $M$ being a fixed integer and other coefficients set to zero. Under the zero-forcing constraints in (35), it is desirable to choose the precoder coefficients such that the correspond set of coefficients $\left\{\mathcal{J}_{u, k, m} \mid 1 \leq u, k \leq U,-M \leq m \leq M\right\}$ are significant. Then applying Property (B4) of Bessel functions in Appendix A gives that

$$
M=2 \pi \min _{u, k}\left\lfloor\frac{\left|X_{u}-X_{k}\right|}{\lambda}\right\rfloor
$$

where users are assumed to be separated by distances much larger than a single wavelength, yielding the following fact.

Remark 8 (Degrees of freedom). Given MU-PM transmission using a circular UA, the total number of degrees of freedom for interference avoidance is approximately equal to $(2 M+1)$ with $M$ given in (36), which is proportional to the minimum user-separation distance.

Transmission over only $(2 M+1)$ phase modes allows the precoder coefficients for user $u$ to be represented by the vector $\mathbf{c}_{u}=\left[\begin{array}{llll}c_{u,-M}, & \cdots, & c_{u, 0}, \cdots, & \cdots, M\end{array}\right]^{T}$. Furthermore, the zero-forcing constraints in (35) can be written in a matrix form:

$$
\mathcal{J}_{u} \mathbf{c}_{u}=\mathbf{0}
$$


where the matrix $\mathcal{J}_{u}$ is defined as

$$
\mathcal{J}_{u}=\left[\begin{array}{ccccc}
\mathcal{J}_{u, 1,-M}, & \cdots, & \mathcal{J}_{u, 1,0}, & \cdots, & \mathcal{J}_{u, 1, M} \\
\vdots & \ddots & \vdots & \ddots & \vdots \\
\mathcal{J}_{u, u-1,-M}, & \cdots, & \mathcal{J}_{u, u-1,0}, & \cdots, & \mathcal{J}_{u, u-1, M} \\
\mathcal{J}_{u, u+1,-M}, & \cdots, & \mathcal{J}_{u, u+1,0}, & \cdots, & \mathcal{J}_{u, u+1, M} \\
\vdots & \ddots & \vdots & \ddots & \vdots \\
\mathcal{J}_{u, U,-M}, & \cdots, & \mathcal{J}_{u, U, 0}, & \cdots, & \mathcal{J}_{u, U, M}
\end{array}\right]
$$

with the elements specified in Lemma 4. Given the constraints obtained in (37), the main result of the section can be readily stated as follows.

Theorem 4 (Multiuser UA precoding). For MU-PM transmission using the circular UA, the precoder coefficients are given as

$$
\mathbf{c}_{u} \in \operatorname{null}\left(\mathcal{J}_{u}\right), \quad u=1,2, \cdots, U
$$

where the matrix $\mathcal{J}_{u}$ is given in (38) and $\operatorname{null}\left(\mathcal{J}_{u}\right)$ denotes the null-space of $\mathcal{J}_{u}$.

With interference avoided, the receive SNR for user $u$ follows from (32) as

$$
\mathrm{SNR}_{u}=\frac{\lambda P_{t}\left|c_{u, 0}\right|^{2}}{8 \pi r_{0} \sigma^{2}}+\frac{o}{r_{0}}=\frac{\lambda P_{t}\left|\mathbf{e}_{0}^{\dagger} \mathbf{c}_{u}\right|^{2}}{8 \pi r_{0} \sigma^{2}}+\frac{o}{r_{0}}
$$

where $\mathbf{e}_{0}=[0, \cdots, 0,1,0, \cdots, 0]^{T}$. Thus, to maximize $\mathrm{SNR}_{u}$, the precoder-coefficient vector $\mathbf{c}_{u}$ should be chosen as the projection of $\mathbf{e}_{0}$ onto $\operatorname{null}\left(\mathcal{J}_{u}\right)$ that contains $\mathbf{c}_{u}$ according to Theorem 4 . This gives the following corollary.

Corollary 2. For MU-PM transmission using the circular UA, the maximum receive SNRs are given as

$$
\mathrm{SNR}_{u}=\frac{\lambda P_{t}\left|\mathbf{e}_{0}^{\dagger} \mathbf{b}_{u}\right|^{2}}{8 \pi r_{0} \sigma^{2}}+\frac{o}{r_{0}}, \quad u=1,2, \cdots, U
$$

where $\mathbf{b}_{u}$ is a basis of $\operatorname{null}\left(\mathcal{J}_{u}\right)$.

With respect to the case of channel-conjugate transmission, the term $\left|\mathbf{e}_{0}^{\dagger} \mathbf{b}_{u}\right|^{2}$ represents the loss in receive SNR due to interference nulling.

\section{Communication Using the Spherical UA}

In the preceding section, the UA is modeled as the circular array. The results are extended in this section to the spherical UA system, which shows its performance improvements with respect to the circular-UA counterpart. Essentially, the difference in analysis arises from the use of spherical harmonics as the tool in place of Fourier series. 


\section{A. Spherical LI-Channel Estimation}

Assume that users transmit single pilot symbols: $\left\{s_{u}\right\}=\{1\}$. The estimation scheme in Section III is extended to the spherical UA as follows. To this end, the training signal in (4), re-denoted as $q(\varphi, \theta)$, is expanded as a Laplace series as follows by using the harmonic functions $\left\{Y_{\ell}^{m}\right\}$, defined in (74) in Appendix A, as the basis:

$$
q(\varphi, \theta)=\sum_{\ell=0}^{\infty} \sum_{m=-\ell}^{\ell} Q_{\ell}^{m} Y_{\ell}^{m}(\varphi, \theta), \quad \theta \in[0, \pi), \varphi \in[0,2 \pi)
$$

where the Laplace coefficients $\left\{Q_{\ell}^{m}\right\}$ are defined as

$$
Q_{\ell}^{m}=\sum_{u=1}^{U} \int_{\theta=0}^{2 \pi} \int_{\psi=0}^{\pi} q(\varphi, \theta) Y_{\ell}^{m}(\varphi, \theta) \sin \varphi d \varphi d \theta .
$$

To derive a closed-form expression for $Q_{\ell}^{m}$, a useful result is given as follows.

Lemma 5. Given two points $\left(r_{0}, \varphi, \theta\right),\left(r_{u}, \varphi_{u}, \theta_{u}\right) \in \mathbb{R}^{3}$,

$$
e^{j \frac{2 \pi}{\lambda} r_{u} \cos \psi_{u}}=(2 \pi)^{\frac{3}{2}} \sum_{\ell=0}^{\infty} \sum_{m=-\ell}^{\ell} \frac{j^{\ell} J_{\ell+\frac{1}{2}}\left(\frac{2 \pi}{\lambda} r_{u}\right)}{\left(\frac{2 \pi}{\lambda} r_{u}\right)^{\frac{1}{2}}} Y_{\ell}^{m}\left(\varphi_{u}, \theta_{u}\right) Y_{\ell}^{m}(\varphi, \theta)
$$

where $\psi_{u}$ denotes the angle between the vectors corresponding to the two points.

The lemma is proved in Appendix B-C. Using the orthogonality of the basis functions $\left\{Y_{\ell}^{m}(\varphi, \theta)\right\}$, substitution of the expression for $q(\varphi, \theta)$ in (4) and Lemma 5 into (43) leads to the following lemma.

Lemma 6 (Training signal decomposition). The training signal received at the spherical UA, namely $\{q(\varphi, \theta)\}$ in (42), has the Laplace coefficients given as follows:

$$
Q_{\ell}^{m}=\frac{\lambda(2 \pi)^{\frac{3}{2}}}{4 \pi r_{0}} \sum_{u=1}^{U} \frac{j^{\ell} J_{\ell+\frac{1}{2}}\left(2 \pi r_{u} / \lambda\right)}{\left(2 \pi r_{u} / \lambda\right)^{\frac{1}{2}}} Y_{\ell}^{m}\left(\varphi_{u}, \theta_{u}\right)+\frac{o}{r_{0}}
$$

where $\ell=1,2, \cdots$ and $-\ell \leq m \leq \ell$.

Define two sets $\tilde{\mathbf{Q}}$ and $\tilde{\mathbf{V}}(Y)$ as $\tilde{\mathbf{Q}}=\left\{Q_{\ell}^{m}\right\}$ and $\tilde{\mathbf{V}}(Y)=\left\{V_{\ell}^{m}(Y)\right\}$ with their elements $Q_{\ell}^{m}$ given in (45) and $V_{\ell}^{m}: \mathbb{R}^{3} \rightarrow \mathbb{R}$ being a function defined as

$$
V_{\ell}^{m}(Y)=(2 \pi)^{\frac{3}{2}} \frac{j^{n} J_{\ell+\frac{1}{2}}\left(\frac{2 \pi}{\lambda} r_{u}\right)}{\left(\frac{2 \pi}{\lambda} r_{u}\right)^{\frac{1}{2}}} Y_{\ell}^{m}\left(\varphi_{u}, \theta_{u}\right) .
$$

Using these definitions, the channel observation profile corresponding to the spherical UA, represented by $\tilde{\Phi}(Y)$, can be defined similarly as in (11):

$$
\begin{aligned}
\tilde{\Phi}(Y) & =\frac{r_{0}}{\lambda}|\tilde{\mathbf{V}}(Y) \circ \tilde{\mathbf{Q}}| \\
& =\frac{r_{0}}{\lambda}\left|\sum_{u=1}^{U} \sum_{\ell=0}^{\infty} \sum_{m=-\ell}^{\ell}\left[V_{\ell}^{m}(Y)\right]^{*} Q_{\ell}^{m}\right| .
\end{aligned}
$$


Substituting (45) and (46) gives

$$
\tilde{\Phi}(Y)=\left|2 \pi^{2} \sum_{u=1}^{U} \sum_{\ell=0}^{\infty} \frac{J_{\ell+\frac{1}{2}}\left(\frac{2 \pi r_{u}}{\lambda}\right) J_{\ell+\frac{1}{2}}\left(\frac{2 \pi r_{Y}}{\lambda}\right)}{\left(\frac{2 \pi r_{u}}{\lambda}\right)^{\frac{1}{2}}\left(\frac{2 \pi r_{Y}}{\lambda}\right)^{\frac{1}{2}}} \sum_{m=-\ell}^{\ell}\left[Y_{\ell}^{m}(Y)\right]^{*} Y_{\ell}^{m}\left(X_{u}\right)\right|+o
$$

where noise varnishes for the same reason as for the case of circular UA. By applying Addition Theorem in Property (S3) for spherical harmonics in Appendix A,

$$
\tilde{\Phi}(Y)=\left|\frac{\pi}{2} \sum_{u=1}^{U} \sum_{\ell=0}^{\infty} \frac{(2 \ell+1) J_{\ell+\frac{1}{2}}\left(\frac{2 \pi r_{u}}{\lambda}\right) J_{\ell+\frac{1}{2}}\left(\frac{2 \pi r_{Y}}{\lambda}\right)}{\left(\frac{2 \pi r_{u}}{\lambda}\right)^{\frac{1}{2}}\left(\frac{2 \pi r_{Y}}{\lambda}\right)^{\frac{1}{2}}} P_{\ell}\left(\cos \psi_{u}\right)\right|^{2}+o .
$$

Next, applying Addition Theorem in Property (B3) of Bessel functions in Appendix A further simplifies the expression as shown in the following theorem.

Theorem 5 (Channel observation). The channel observation profile corresponding to the spherical UA is given as

$$
\tilde{\Phi}(Y)=\left|\sum_{u=1}^{U} \operatorname{sinc}\left(\frac{2 \pi}{\lambda}\left|Y-X_{u}\right|\right)\right|+o, \quad \text { a.s. }
$$

Remark 9 (Channel estimation error). As in Remark 2 for the circular UA, the accuracy of channel estimation can be evaluated using the difference $\left|\tilde{\Phi}\left(X_{u}\right)-1\right|$. Since $|\operatorname{sinc}(d)| \leqslant d^{-1}$, it can be obtained from Theorem 5 that

$$
\left|\tilde{\Phi}\left(X_{u}\right)-1\right| \leq 2 \pi(U-1) \min _{u \neq k}\left(\frac{\left|X_{k}-X_{u}\right|}{\lambda}\right)^{-1}+o, \quad \text { a.s. }
$$

The error bound is observed to diminish inversely with the increasing minimum user-separation distance (in wavelength) following an inverse function that is faster than the circular-UA counterpart in (13). This quantifies the gain of increasing the UA by one dimension from the perspective of channel estimation.

Next, consider the case where users transmit pilot sequences with length of $L$ symbols. Following the same procedure as for deriving Theorem 2 yields the following corollary.

Corollary 3 (Effect of pilot sequences). Given $L \geq U$ and orthogonal pilot sequences, the LI-channel estimation using the spherical UA is almost perfect since the channel observation profile is approximately equal to the single-user counterpart:

$$
\left|\tilde{\Phi}_{u}(Y)-\operatorname{sinc}\left(\frac{2 \pi}{\lambda}\left|X_{u}-Y\right|\right)\right| \leqslant o .
$$

Remark 10. If orthogonal pilot sequences are used, the spherical UA does not have an advantage over the circular counterpart in terms of channel estimation. However, the former improves the performance of channel estimation in the case of single pilot symbols as well as that of data communication as shown in the sequel. 


\section{B. Data Transmission Using the Spherical UA}

1) Channel Conjugate Transmission: For channel conjugate transmission using the spherical UA, the spherical precoder, denoted as $\tilde{f}_{u}(\varphi, \theta)$, is modified from the circular counterpart in (20) as

$$
\tilde{f}_{u}(\varphi, \theta)=\frac{h_{u}^{*}(\varphi, \theta)}{\left|h_{u}(\varphi, \theta)\right|}, \quad \theta \in[0,2 \pi), \varphi \in[0, \pi) .
$$

The resultant receive SNRs are derived as follows. Let $\tilde{q}\left(X_{u} \mid X_{k}\right)$ and $\tilde{p}\left(X_{u} \mid X_{k}\right)$ denote the field and its power density measured at location $X_{u}$ given a precoder targeting user $X_{k}$. Then $\tilde{q}\left(X_{u} \mid X_{k}\right)$ can be obtained by modifying the circular-UA counterpart in (21) by replacing the integration over a circle with one over a sphere:

$$
\begin{aligned}
\tilde{g}\left(X_{u} \mid X_{k}\right) & =\sqrt{\frac{P_{\mathrm{t}}}{4 \pi r_{0}^{2}}} \times \frac{1}{\sqrt{4 \pi r_{0}^{2}}} \times \iint e^{j \frac{2 \pi}{\lambda}\left(r_{u} \cos \psi_{u}-r_{k} \cos \psi_{k}\right)} r_{0}^{2} \sin \theta d \theta d \varphi+o \\
& =\frac{\sqrt{P_{\mathrm{t}}}}{4 \pi} \iint e^{j \frac{2 \pi}{\lambda}\left(r_{u} \cos \psi_{u}-r_{k} \cos \psi_{k}\right)} \sin \theta d \theta d \varphi+o .
\end{aligned}
$$

To facilitate analysis, a set of coefficients $\left\{\mathcal{J}_{u, k, m, n}\right\}$ with integer indices $(u, k, m, n)$ are defined as

$$
\mathcal{J}_{u, k, m, \ell}=\iint e^{j \frac{2 \pi}{\lambda}\left(r_{u} \cos \psi_{u}-r_{k} \cos \psi_{k}\right)} Y_{\ell}^{m}(\varphi, \theta) \sin \varphi d \varphi d \theta
$$

with $1 \leq u, k \leq U, n \geq 0$ and $-\ell \leq m \leq \ell$, which are also used for designing multiuser precoders in the next sub-section. They can be written in a closed form as shown in the following lemma that is proved in Appendix B-D.

Lemma 7. The coefficient $\mathcal{J}_{u, k, m, \ell}$ defined in (53) can be written as

$$
\mathcal{J}_{u, k, m, \ell}=\frac{(2 \pi)^{\frac{3}{2}} j^{\ell} J_{\ell+\frac{1}{2}}\left(\frac{2 \pi}{\lambda}\left|X_{u}-X_{k}\right|\right)}{\sqrt{\frac{2 \pi}{\lambda}\left|X_{u}-X_{k}\right|}} Y_{\ell}^{m}\left(\varphi_{u, k}, \theta_{u, k}\right)
$$

where the angles $\varphi_{u, k}$ and $\theta_{u, k}$ are defined by the following equations:

$$
\begin{aligned}
\sin \varphi_{u, k} \cos \theta_{u, k} & =\frac{r_{u} \sin \varphi_{u} \cos \theta_{u}-r_{k} \sin \varphi_{k} \cos \theta_{k}}{r_{u, k}} \\
\sin \varphi_{u, k} \sin \theta_{u, k} & =\frac{r_{u} \sin \varphi_{u} \sin \theta_{u}-r_{k} \sin \varphi_{k} \sin \theta_{k}}{r_{u, k}} \\
\cos \varphi_{u, k} & =\frac{r_{u} \cos \varphi_{u}-r_{k} \cos \varphi_{k}}{r_{u, k}} .
\end{aligned}
$$

Using the fact that

$$
J_{\frac{1}{2}}(x)=\sqrt{\frac{2}{\pi}} \frac{\sin x}{\sqrt{x}}, \quad Y_{0,0}\left(\varphi_{u, k}, \theta_{u, k}\right)=\frac{1}{\sqrt{4 \pi}}
$$

and Lemma 7, the field in (52) can be obtained in a closed form, yield the following result.

Lemma 8 (Field power density distribution). Given the spherical UA and channel conjugate precoding targeting user $X_{u}$, the field power density measured at the user location $X_{k}$ is given as

$$
\tilde{p}\left(X_{u} \mid X_{k}\right)=P_{t} \operatorname{sinc}^{2}\left(\frac{2 \pi}{\lambda}\left|X_{u}-X_{k}\right|\right)+o .
$$


As in the case of circular UA, $\tilde{p}\left(X_{u} \mid X_{k}\right)$ can be rewritten as $\tilde{p}(d)$ with $d$ being the distance from the location targeted by the precoder. Then the distribution of the field power density can be characterized by the function $\tilde{p}(d) / \tilde{p}(0)=\operatorname{sinc}^{2}\left(\frac{2 \pi d}{\lambda}\right)$ that is plotted in Fig. 3. Like the circular-UA counterpart, by channel-conjugate precoding, the spherical UA focuses the transmission power into a region within a distance of half wavelength from the target location. The advantage of the spherical UA is reflected in that the tail of the distribution function has an envelop diminishing with the increasing distance much faster than that corresponding to the circular UA. This reduces multiuser interference and leads to substantial performance improvements as shown in the analysis and observed from simulation results.

Given the SINR defined similarly as in (25), the result in Lemma 8 leads to the spherical-UA counterpart of Theorem 3 as follows.

Theorem 6 (Receive SINRs). For channel conjugate transmission using the spherical UA, the receive SINR for user $u$, denoted as $\widetilde{\mathrm{SINR}}$ is given as

$$
\widetilde{\operatorname{SINR}}_{u}=\frac{1}{\sum_{k \neq u} \operatorname{sinc}^{2}\left(\frac{2 \pi}{\lambda}\left|X_{u}-X_{k}\right|\right)+\frac{1}{\operatorname{SNR}}}+o, \quad u=1,2, \cdots, U
$$

where the receive $S N R$ is

$$
\mathrm{SNR}=\frac{P_{t} \lambda^{2}}{4 \pi \sigma^{2}}
$$

Remark 11 (High SNR). Using the fact $|\operatorname{sinc}(d)| \leqslant d^{-1}$, the SIR lower bound in (28) for the circular UA can be modified for the current case as

$$
\widetilde{\operatorname{SIR}}_{u} \geqslant \frac{1}{U-1}\left(2 \pi \min _{k \neq u} \frac{\left|X_{k}-X_{u}\right|}{\lambda}\right)^{2}+o .
$$

Thus, the receive SIRs increase with the minimum user-separation distance (in wavelength) at least following a super-linear function with the exponent 2 , which is faster than the sub-linear function for the circular UA (see Remark 5). This specifies the gain of increasing the UA by one dimension from the perspective of received signal quality.

Last, the received SNR in (58) suggests the following result.

Corollary 4 (Propagation loss). Given channel-conjugate transmission using the spherical UA, the propagation loss is approximately equal to the receive antenna aperture:

$$
\frac{P_{\mathrm{r}}}{P_{\mathrm{t}}}=\frac{\lambda^{2}}{4 \pi}+o
$$

In other words, the path loss is a constant and independent with the propagation distance $r_{0}$. In contrast, the loss corresponding to the circular UA and the conventional array is inversely proportional to $r_{0}$ (see Corollary 1) and $r_{0}^{2}$, respectively. 
2) Multiuser Phase Mode Transmission: The phase modes for the spherical UA correspond to different spherical harmonics. Then the spherical MU-PM precoder for user $u$, denoted as $\tilde{f}_{u}^{\prime}$, is modified from the circular counterpart in (31) as

$$
\tilde{f}_{u}^{\prime}(\varphi, \theta)=\tilde{f}_{u}(\varphi, \theta) \sum_{\ell=0}^{\infty} \sum_{m=-\ell}^{\ell} c_{u, m, \ell} Y_{\ell}^{m}(\varphi, \theta)
$$

where $\left\{c_{u, m, \ell}\right\}$ are the precoder coefficients to be designed, $\tilde{f}_{u}(\varphi, \theta)$ is the channel conjugate precoder in (51) and the spherical harmonic function $Y_{\ell}^{m}(\varphi, \theta)$ is defined in (74). By substitution of (61) into (5), the signal received at user $X_{u}$ is given as

$$
\begin{aligned}
\tilde{y}_{u}= & \lambda \sqrt{\frac{P_{t}}{4 \pi}} \sum_{k=1}^{U}\left(\frac{1}{4 \pi} \iint \frac{h_{k}^{*}(\varphi, \theta) h_{u}(\varphi, \theta)}{\left|h_{k}(\varphi, \theta)\right|} \sum_{\ell=0}^{\infty} \sum_{m=0}^{\ell} c_{k, m, \ell} Y_{\ell}^{m}(\varphi, \theta) \sin \varphi d \varphi d \theta\right) x_{k}+z_{u} \\
= & \lambda \sqrt{\frac{P_{t}}{4 \pi}} c_{u, 0,0} x_{u}+\lambda \sqrt{\frac{P_{t}}{4 \pi}} \sum_{k \neq u}\left(\frac{1}{4 \pi} \iint e^{j \frac{2 \pi}{\lambda}\left(r_{u} \cos \psi_{u}-r_{k} \cos \psi_{k}\right)} \sum_{\ell=0}^{\infty} \sum_{m=-\ell}^{\ell} c_{k, m, \ell} Y_{\ell}^{m}(\varphi, \theta) \sin \varphi d \varphi d \theta\right) x_{k} \\
& +z_{u}+o \\
= & \lambda \sqrt{\frac{P_{t}}{4 \pi}} c_{u, 0,0} x_{u}+\lambda \sqrt{\frac{P_{t}}{4 \pi}} \sum_{k \neq u} \sum_{\ell=0}^{\infty} \sum_{m=0}^{\ell} c_{k, m, \ell} \mathcal{J}_{u, k, m, \ell} x_{k}+z_{u}+o
\end{aligned}
$$

where the coefficients $\left\{\mathcal{J}_{u, k, m, \ell}\right\}$ are defined earlier in (53). It can be observed from Lemma 7 that $\mathcal{J}_{u, k, m, \ell}$ is proportional to $J_{\ell+\frac{1}{2}}\left(\frac{2 \pi}{\lambda}\left|X_{u}-X_{k}\right|\right)$. Therefore, following the same reason as for the circular $\mathrm{UA}$, only the set of coefficients $\left\{\mathcal{J}_{u, k, m, \ell} \mid 0 \leq \ell \leq M\right\}$ have significant values. Considering only these values reduces the precoder coefficients to a finite set $\left\{c_{k, m, \ell} \mid 0 \leq \ell \leq M\right\}$, yielding the following fact.

Remark 12 (Degrees of freedom). Given MU-PM transmission using a spherical UA, the total number of degrees of freedom for interference avoidance is approximately equal to $(M+1)^{2}$ with $M$ given in (36). As a result, since $M$ is much greater than one, the number of degrees of freedom generated by the spherical UA is approximately proportional to $M^{2}$ that is much larger than that, namely $2 M$, for the circular counterpart.

Next, the said finite set of precoder coefficients can be designed by applying the zero-forcing constraints that follow from (62) as

$$
\sum_{\ell=0}^{M} \sum_{m=-\ell}^{\ell} c_{k, m, \ell} \mathcal{J}_{u, k, m, \ell}=0, \quad \forall k \neq u .
$$


The constraints can be written in the matrix form. To this end, define the matrix $\mathcal{H}_{u, \ell}$ as

$$
\mathcal{H}_{u, \ell}=\left[\begin{array}{cccc}
\mathcal{J}_{u, 1,-\ell, \ell}, & \mathcal{J}_{u, 1,-\ell+1, \ell}, & \cdots & \mathcal{J}_{u, 1, \ell, \ell} \\
\vdots & \vdots & \ddots & \vdots \\
\mathcal{J}_{u, u-1,-\ell, \ell}, & \mathcal{J}_{u, u-1,-\ell+1, \ell}, & \cdots & \mathcal{J}_{u, u-1, \ell, \ell} \\
\mathcal{J}_{u, u+1,-\ell, \ell}, & \mathcal{J}_{u, u+1,-\ell+1, \ell}, & \cdots & \mathcal{J}_{u, u+1, \ell, \ell} \\
\vdots & \vdots & \ddots & \vdots \\
\mathcal{J}_{u, U,-\ell, \ell}, & \mathcal{J}_{u, U,-\ell+1, \ell}, & \cdots & \mathcal{J}_{u, U, \ell, \ell}
\end{array}\right]
$$

and the row vector $\mathbf{a}_{u, \ell}=\left[\begin{array}{llll}c_{u,-\ell, \ell}, & c_{u,-\ell+1, \ell}, & \cdots & c_{u, \ell, \ell}\end{array}\right]$ where $\ell=0,1, \cdots$. Moreover, using these matrices/vectors as elements, define

$$
\tilde{\mathcal{J}}_{u}=\left[\mathcal{H}_{u, 0}, \mathcal{H}_{u, 2}, \cdots, \mathcal{H}_{u, M}\right], \quad \mathbf{c}_{u}=\left[\mathbf{a}_{u, 1}, \mathbf{a}_{u, 2}, \cdots, \mathbf{a}_{u, \widetilde{M}}\right]^{T} .
$$

Using these definitions, the zero forcing constraints in (63) can be written as

$$
\tilde{\mathcal{J}}_{u} \tilde{\mathbf{c}}_{u}=\mathbf{0}, \quad u=1,2, \cdots, U
$$

The main result of the section is summarized in the following theorem.

Theorem 7 (Multiuser UA precoding). For MU-PM transmission using the circular UA, the precoder coefficients under the zero-forcing constraints are given as

$$
\tilde{\mathbf{c}}_{u} \in \operatorname{null}\left(\tilde{\mathcal{J}}_{u}\right), \quad u=1,2, \cdots, U
$$

where $\operatorname{null}\left(\tilde{\mathcal{J}}_{u}\right)$ denotes the null-space of $\tilde{\mathcal{J}}_{u}$.

The spherical-UA counterpart of Corollary 2 is as follows.

Corollary 5 (Receive SNRs). For MU-PM transmission using the circular UA, the maximum receive SNR for user $u$ is given as

$$
\mathrm{SNR}_{u}=\frac{\eta^{2} P_{\mathrm{t}}\left|\mathbf{e}_{0}^{\dagger} \mathbf{b}_{u}\right|^{2}}{\sigma^{2}}+o, \quad u=1,2, \cdots, U
$$

where $\mathbf{b}_{u}$ is a basis of $\operatorname{null}\left(\tilde{\mathcal{J}}_{u}\right)$.

Remark 13. Theorem 7 and Corollary 5 are observed to have the same forms as Theorem 3 and Corollary 2 , respectively. However, the space $\operatorname{null}\left(\tilde{\mathcal{J}}_{u}\right)$ corresponding to the sperical UA is much larger than its circular-UA counterpart null $\left(\mathcal{J}_{u}\right)$. The extra degrees of freedom allow the spherical-UA system to support a larger number of simultaneous users and reduce the received SNR loss due to interference avoidance. 


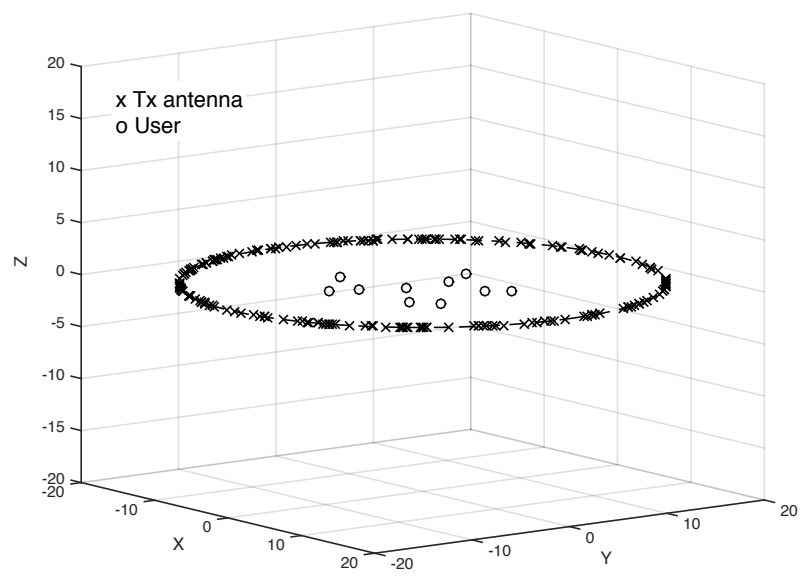

(a) Circular UA

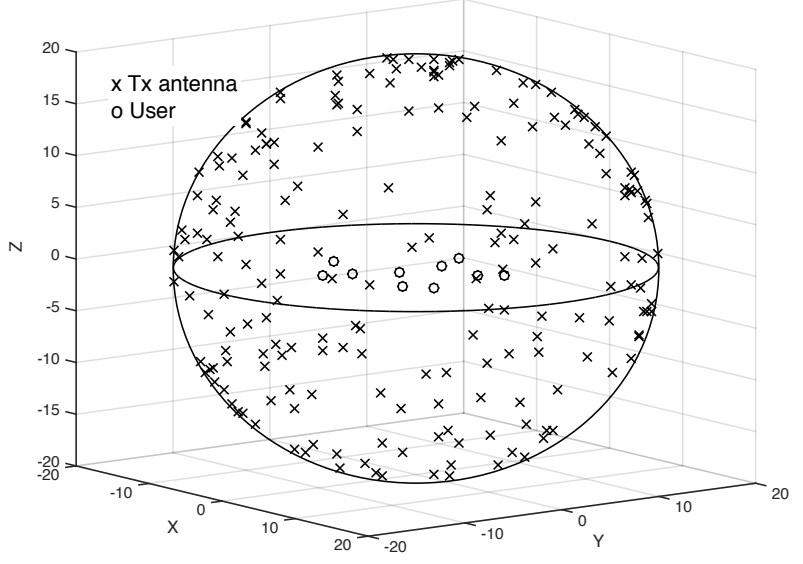

(b) Spherical UA

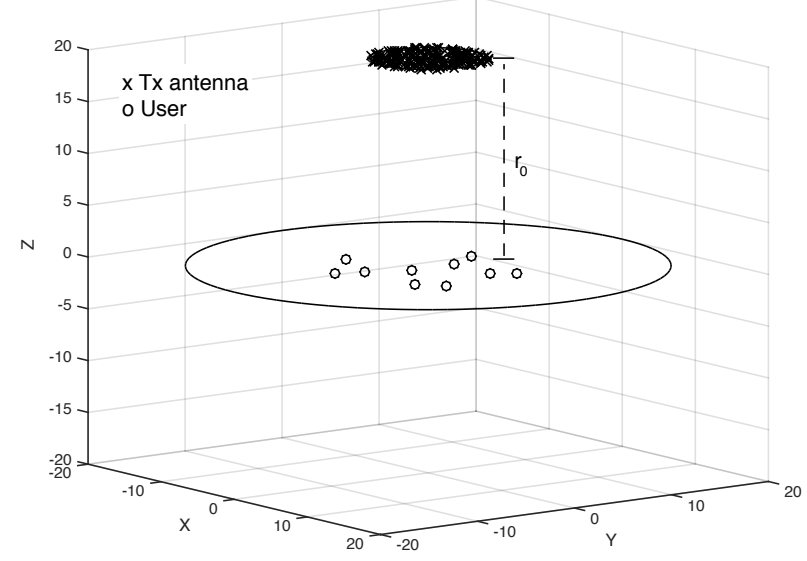

(c) Collocated array

Fig. 4. (a) Circular UA with a radius of $r_{0}=20 \mathrm{~m}$. (b) Spherical UA with the same radius. (c) Collocated array with a radius of $r_{d}=5 \mathrm{~m}$. The number of users is 10 .

\section{Simulation Results}

In simulation, discrete UAs with finite numbers of antennas are considered. As illustrated in Fig. 4, the UA antennas are uniformly distributed on a circle for the case of circular UA or a sphere for the case of spherical UA, both of which have the fixed radius $r_{0}=20 \mathrm{~m}$ modeling a small deployment region. For benchmarking, the conventional collocated array is also included in simulation whose antennas are uniformly distributed in a horizontal disk with a radius denoted as $r_{d}$ and right above the origin with a vertical distance equal to $r_{0}$ as shown in Fig. 4(c). Note that this location and orientation of the collocated UA are found by simulation to yield the best performance among other configurations with the same disk radius and distance to the origin. The radius of the collocated UA is set as $r_{d}=5 \mathrm{~m}$ in Fig. 4 for ease of illustration and $r_{d}=1 \mathrm{~m}$ for all other simulation results. In addition, relaxing Assumption 1, the users are uniformly distributed in the horizontal disk with a radius of $0.5 r_{0}$ instead of being near the origin. Moreover, the carrier frequency is $2.5 \mathrm{GHz}$ and the noise variance is $-100 \mathrm{dBm}$. 


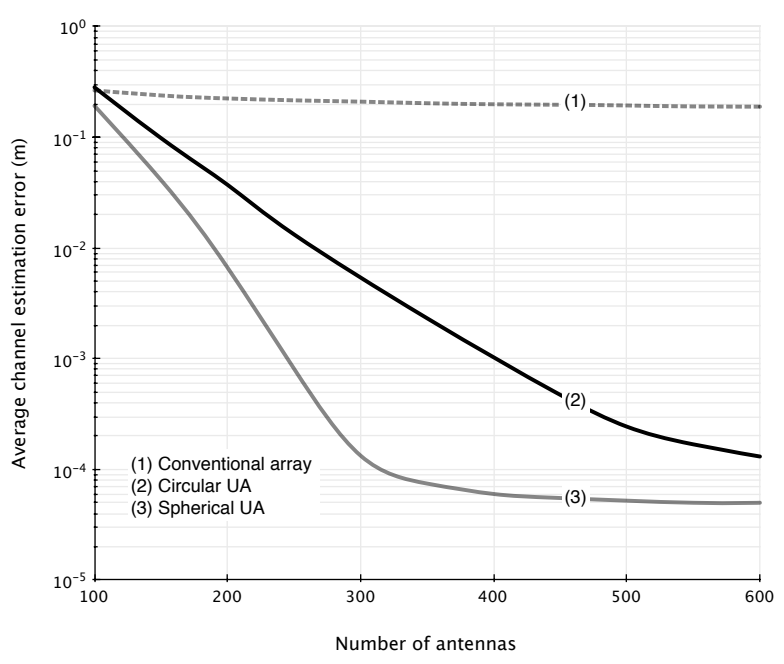

(a) Effect of the number of antennas

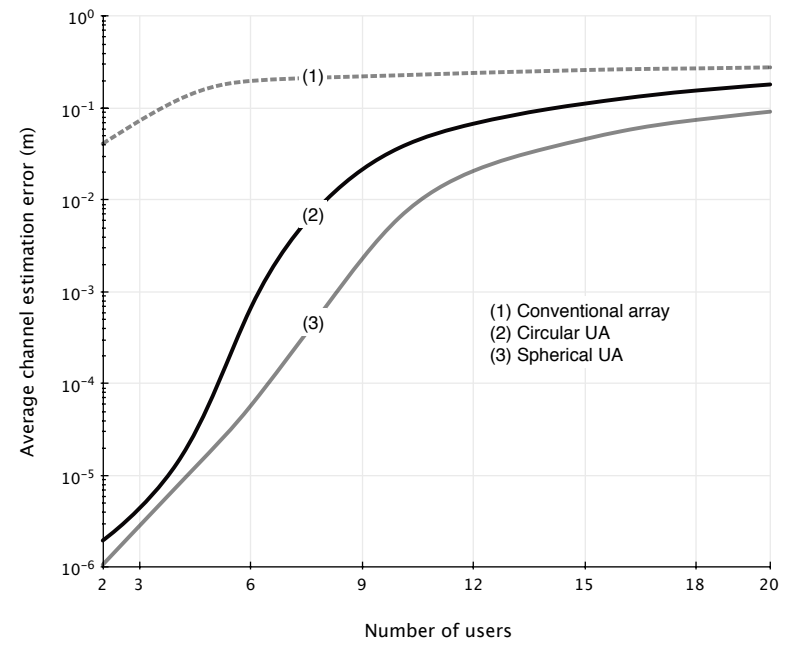

(b) Effect of the number of users

Fig. 5. Consider LI-channel estimation with single pilot symbols. (a) Effect of the number of antennas on channel estimation error for 10 users. (b) Effect of the number of users on channel estimation error for 200 transmit antennas.

\section{A. Channel Estimation}

Consider channel estimation using single pilot symbols and algorithms from straightforward extension of those in Sections III and V-A to discrete arrays. The average channel estimation error is defined as the difference between the estimated and actual locations of a typical user as averaged over the random distributions of users and antennas. Fig. 5 displays the curves of average channel estimation error versus the number of antennas and those of average error versus the number of users in two separate sub-figures. Several observations can be made from the curves. As the number of antennas increases, the average errors for the circular and spherical UAs both diminishes rapidly and converges to a small constant corresponding to the continuous arrays analyzed in the preceding sections. Moreover, the errors grows rapidly as the number of users increases. For relatively small numbers of users (e.g, fewer than 9) or large numbers of antennas (e.g., larger than 200), the performance of channel estimation for the UAs is much better than that using a collocated UA; the spherical UA outperforms the circular UA. The conventional array's incapability of accurate LI-channel estimation is mainly due to its confined geometry that is more suitable for estimating signals' angles-of-arrival. For verification, it can observed from Fig. 5 that the average error for the collocated array is insensitive to the numbers of antennas and users, suggesting that they are not the performance limiting factors.

\section{B. Data Transmission}

Consider data transmission assuming perfect channel-state-information at the transmitter. The curves of sum throughput versus transmission power per user are plotted in Fig. 6 with the number of users fixed at 10. The plots account for two transmission schemes including multiuser phase-mode (PM) and single-user channel-conjugate (CC) transmission and different numbers of antennas, namely 100 and 400. Several observations are made by comparing the curves. First, the combination of spherical UA and PM 


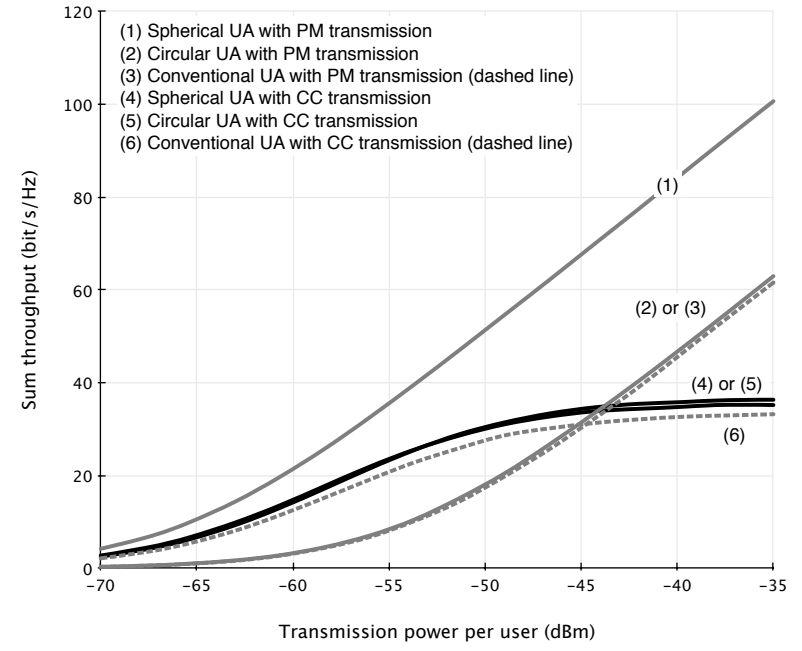

(a) Transmit array with 100 antennas

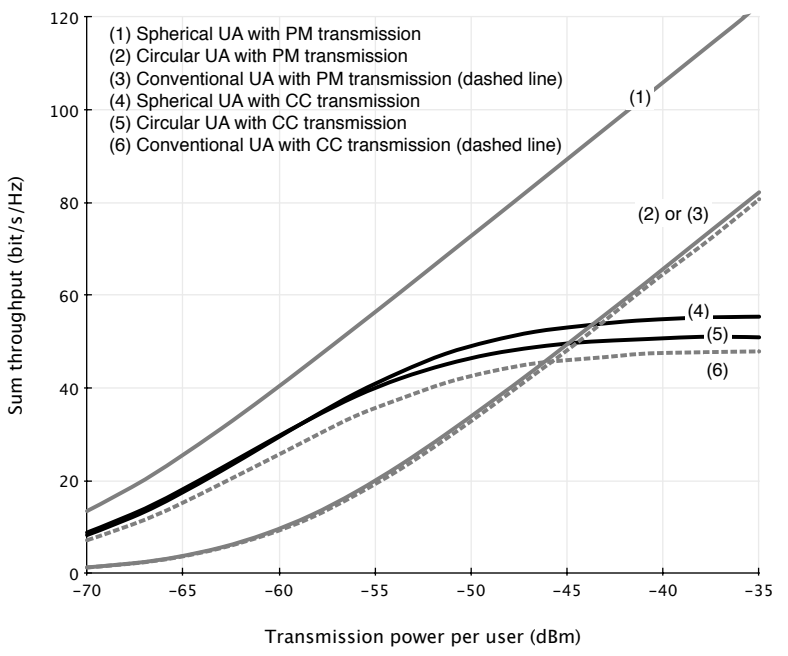

(b) Transmit array with 400 antennas

Fig. 6. Sum throughput versus transmission power per user for a transmit array with (a) 100 or (b) 400 antennas. The number of users is fixed at 10 .

transmission yields much higher sum throughput than any other combination since distributing antennas over a larger area allows the spherical UA to generate a much higher number degrees of freedom for avoiding interference and enhancing received signal power compared with other arrays (see Remark 12). Second, the sum throughputs for CC transmission using three types of arrays are comparable and higher than those achieved by PM transmission using the circular UA and collocated UA in the power range of -70 to $-45 \mathrm{dBm}$; beyond this range, interference resulting from $\mathrm{CC}$ transmission dominates noise, causing the corresponding sum throughputs to saturate. Last, increasing the number of antennas from 100 to 400 contributes approximately the same throughput gain, about $20 \mathrm{~b} / \mathrm{s} / \mathrm{Hz}$, for different combinations of array and transmission scheme.

The curves of sum throughput versus number of users are plotted in Fig. 7 with the transmission power per user fixed at $-40 \mathrm{dBm}$. For PM transmission, the sum throughputs are observed to increase approximately linearly with the increasing number of users or equivalently the increasing number of simultaneous data streams. Furthermore, the curve corresponding to the spherical UA has a slope much larger than those for the other types of arrays that are comparable. In contrast, operating in the interference limiting regime, the sum throughputs for CC transmission using 100 antennas saturate and are observed to be insensitive to the increase of the number of users. This issue can be alleviated by deploying more antennas (400) that leads to a substantial throughput gain e.g., about $20 \mathrm{~b} / \mathrm{s} / \mathrm{Hz}$ for the number of users equal to 18. Nevertheless, the gains for PM transmission are much larger and about $60 \mathrm{~b} / \mathrm{s} / \mathrm{Hz}$ at the same number of users.

\section{CONCLUSION}

Techniques have been designed for channel estimation and data transmission in the UA system by modeling the UA as a gigantic continuous circular/spherical array and assuming free-space propagation. It 


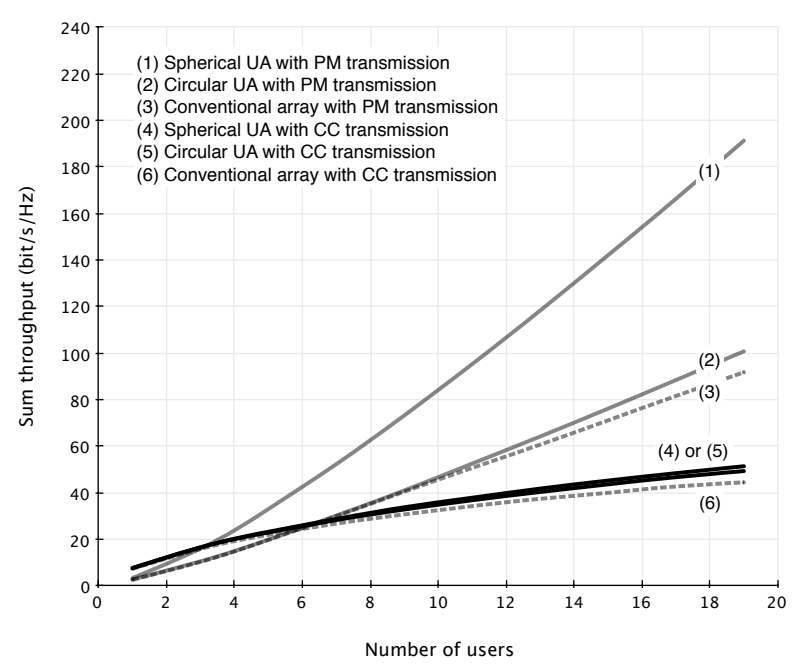

(a) UA with 100 antennas

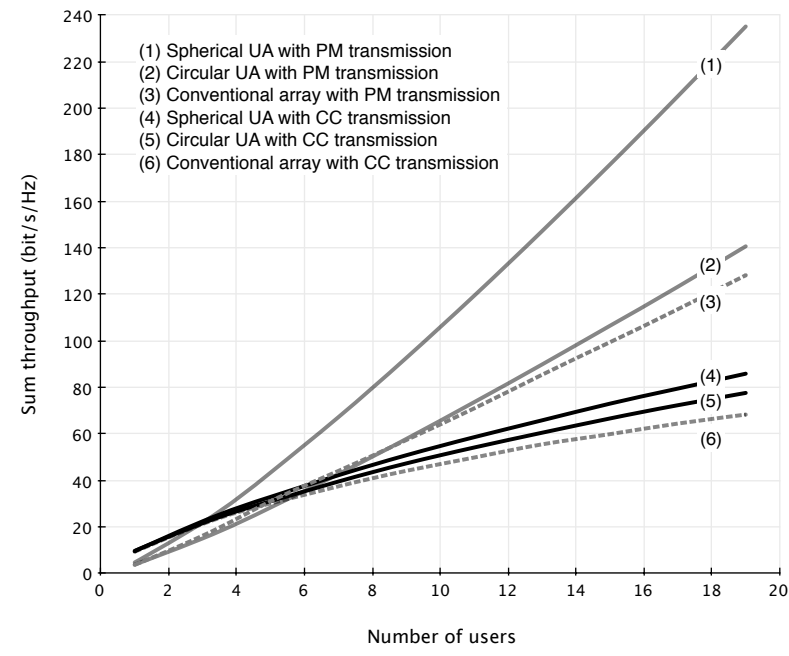

(b) UA with 400 antennas

Fig. 7. Sum throughput versus number of users for a transmit array with (a) 100 or (b) 400 antennas. The transmission power per users is fixed at $-40 \mathrm{dBm}$.

has been shown that the UA enables accurate estimation of multiuser channels even if single pilot symbols are used provided that the user-separation distances are sufficiently large. If orthogonal pilot sequences are used, channel estimation is found to be always close to perfect. For single-user data transmission using the UA, inter-user interference can be suppressed by increasing user-separation distances. Alternatively, interference can be nulled at the UA based a novel design of multiuser phase-mode precoders. The resultant number of available degrees of freedom for interference nulling is shown to be proportional to the minimum user-separation distance. Furthermore, the spherical UA provides performance gain compared with the circular counterpart.

This work opens up several interesting directions for further research. First, the current analysis is based on the model of a continuous circular/spherical UA and targets users near the UA center. Generalizing the model and user locations makes the analysis more challenge and requires the development of new analytical techniques. Second, it is important to address practical issues in the design and analysis of UA communication techniques such as delay and error in message exchange between the UA elements and the presence of sparse scatterers. Furthermore, it is also interesting to design algorithms/protocols for resource allocation, broadband transmission, and power control for the UA systems.

\section{APPENDIX A}

\section{Mathematics Preliminary: Bessel Functions And Spherical Harmonics}

Bessel functions and spherical harmonics are extensively used in the subsequent analysis. In this appendix, the functions are defined and some key properties useful for the analysis are summarized. 


\section{A. Bessel Functions and Their Properties}

Only Bessel functions of the first kind are needed in the analysis and referred to simply as Bessel functions. A Bessel function with an integer order $n$ can be defined in an integral form as [25]:

$$
J_{n}(x)=\frac{1}{2 \pi} \int_{0}^{2 \pi} e^{j(x \sin \varphi-n \varphi)} d \varphi .
$$

Several useful properties of Bessel functions are described as follows.

(B1) The Jacobi-Anger expansion decomposes an exponential function of a trigonometric function into its harmonics as follows [25]:

$$
e^{j x \cos \varphi}=\sum_{n=-\infty}^{\infty} j^{n} J_{n}(x) e^{j k \varphi} .
$$

(B2) Addition Theorem I [26, (6.61)]:

$$
J_{n}(R) e^{j n \omega}=\sum_{k=-\infty}^{\infty} J_{k}(a) J_{n+k}(b) e^{j k \varphi}
$$

where $R=\sqrt{a^{2}+b^{2}-2 a b \cos \varphi}$ and $\sin \omega=(a / R) \sin \varphi$.

(B3) Addition Theorem II rewritten from [26, (6.62)]:

$$
\operatorname{sinc}(R)=\frac{\pi}{2} \sum_{n=0}^{\infty}(2 n+1) \frac{J_{n+\frac{1}{2}}(a) J_{n+\frac{1}{2}}(b)}{\sqrt{a b}} P_{n}(\cos \varphi)
$$

where $P_{n}(x)$ with $x \in[-1,1]$ is the Legendre polynomial defined as

$$
P_{n}(x)=\frac{1}{2^{n} n !} \frac{d^{n}}{d x^{n}}\left(x^{2}-1\right)^{n} .
$$

(B4) The Bessel function $J_{0}(x)$ can be upper bounded as [27]

$$
J_{0}(x) \leqslant \nu x^{-\frac{1}{3}}
$$

where the constant $\nu=0.7857 \cdots$.

(B5) Given $0<z \leq 1$, a Bessel function with a high order satisfies [28, 9.3.5 and 9.3.6] ${ }^{1}$

$$
J_{n}(z n) \sim \frac{c(z)}{n^{\frac{1}{3}}}, \quad n \rightarrow \infty
$$

where $c(z)$ is a positive constant whose value depends only on $z$. Consequently, for $x \gg 1$ and $|n| \geqslant x, J_{n}(x) \approx 0[16]$.

(B6) Gegenbauer's generalization of Poisson's integral [28, 10.1.14]:

$$
\sqrt{\frac{\pi}{2 x}} J_{n+\frac{1}{2}}(x)=\frac{1}{2}(-j)^{n} \int_{-1}^{1} e^{j x \tau} P_{n}(\tau) d \tau
$$

where $P_{n}(x)$ is the Legendre polynomial defined in (70).

\footnotetext{
${ }^{1}$ Two functions $f$ and $g$ are asymptotic equivalent, denoted as $f(x) \sim g(x)$, if $\lim _{x \rightarrow \infty} f(x) / g(x)=1$.
} 


\section{B. Spherical Harmonics and Their Properties}

The spherical harmonic functions denoted as $\left\{Y_{\ell}^{m}(\varphi, \theta)\right\}$ with integer indices $\ell=0,1, \cdots$ and $-\ell \leqslant$ $m \leqslant \ell$ are defined as [25]:

$$
Y_{\ell}^{m}(\varphi, \theta)=\sqrt{\frac{2 m+1}{4 \pi} \frac{(\ell-m) !}{(\ell+m) !}} P_{\ell}^{m}(\cos \theta) e^{j m \varphi}, \quad \theta \in[0, \pi], \varphi \in[0,2 \pi]
$$

where $P_{\ell}^{m}(\cos \theta)$ represents the associated Legendre polynomial defined as

$$
P_{\ell}^{m}(x)=\frac{(-1)^{m}}{2^{\ell} \ell !}\left(1-x^{2}\right)^{\frac{m}{2}} \frac{d^{m+\ell}}{d x^{m+\ell}}\left(x^{2}-1\right)^{\ell}, \quad-1 \leq x \leq 1 .
$$

Note that $P_{\ell}^{m}(\cos \varphi)$ with $m=0$ reduces to the Legendre polynomial in (70).

Several useful properties of the spherical harmonics are described as follows.

(S1) The functions $\left\{Y_{\ell}^{m}(\varphi, \theta)\right\}$ are orthonormal over the spherical surface:

$$
\int_{\varphi=0}^{2 \pi} \int_{\theta=0}^{\pi}\left[\left(Y_{\ell}^{m}\right)(\varphi, \theta)\right]^{*} Y_{\ell^{\prime}}^{m^{\prime}}(\varphi, \theta) \sin \theta d \theta d \varphi=\delta_{m, m^{\prime}} \delta_{\ell, \ell^{\prime}}
$$

where $\delta_{m, m^{\prime}}$ is equal to 1 if $m=m^{\prime}$ and 0 otherwise.

(S2) Funk-Hecke Theorem [30, Theorem 3]: Let $\psi_{u}(\varphi, \theta)$ denote the angle between the vectors $X_{u}=$ $\left(r_{u}, \varphi_{u}, \theta_{u}\right)$ and $(1, \varphi, \theta) \in \mathbb{R}^{3}$. The Laplace series of a function $w\left(\cos \psi_{u}(\varphi, \theta)\right)$ is given as

$$
w\left(\cos \psi_{u}(\varphi, \theta)\right)=\sum_{\ell=0}^{\infty} \sum_{m=-\ell}^{\ell} c_{\ell}^{m}\left(\varphi_{u}, \theta_{u}\right) Y_{\ell}^{m}(\varphi, \theta)
$$

where the coefficient $c_{\ell}^{m}\left(\varphi_{u}, \theta_{u}\right)=c_{\ell} Y_{\ell}^{m}\left(\varphi_{u}, \theta_{u}\right)$ with

$$
c_{\ell}=2 \pi \int_{-1}^{1} w(\tau) P_{\ell}(\tau) d \tau
$$

(S3) Spherical Harmonic Addition Theorem [25, (16.57)]:

$$
\sum_{m=-\ell}^{\ell}\left[Y_{\ell}^{m}(A)\right]^{*} Y_{\ell}^{m}\left(X_{u}\right)=\frac{2 \ell+1}{4 \pi} P_{\ell}\left(\cos \psi_{u}(A)\right)
$$

where $A, X_{u} \in \mathbb{R}^{3}$ and $\psi_{u}(A)$ denotes their separation angle.

\section{APPENDIX B}

PROOFS OF LEMMAS

\section{A. Proof of Lemma 1}

By substituting (4) and $\varphi_{u}(A)=\varphi_{u}-\varphi$ into (8),

$$
Q_{k}=\frac{\lambda e^{-j \frac{2 \pi}{\lambda} r_{0}}}{4 \pi r_{0}} \sum_{u=1}^{U} \int_{0}^{2 \pi} e^{j \frac{2 \pi}{\lambda} r_{u} \cos \left(\varphi_{u}-\varphi\right)} e^{j k \varphi} d \varphi+\frac{1}{2 \pi} \int_{0}^{2 \pi} z(\varphi) e^{j k \varphi} d \varphi+\frac{o}{r_{0}}
$$

The noise term can be written as

$$
\frac{1}{2 \pi} \int_{0}^{2 \pi} z(\varphi) e^{j k \varphi} d \varphi=\lim _{N \rightarrow \infty} \frac{1}{N} \sum_{n=1}^{N} z\left(\frac{2 \pi n}{N}\right) e^{j k \frac{2 \pi n}{N}}
$$


For ease of notation, define $\tilde{z}_{n}=z\left(\frac{2 \pi n}{N}\right) e^{j k \frac{2 \pi n}{N}}$. Since $z_{0}, z_{1}, \cdots, \tilde{z}_{N}$ is an i.i.d. sequence of $\mathcal{C N}\left(0, \sigma^{2}\right)$ random variables under Assumption 2, by applying the law of large numbers, it follows from (79) that

$$
\frac{1}{2 \pi} \int_{0}^{2 \pi} z(\varphi) e^{j k \varphi} d \varphi=0, \quad \text { a.s. }
$$

Next, based on the Jacobi-Anger expansion in Property (B1) of Bessel functions in Appendix A, the first exponential term in (79) can be decomposed as

$$
e^{j \frac{2 \pi}{\lambda} r_{u} \cos \left(\varphi_{u}-\varphi\right)}=\sum_{n=0}^{\infty} j^{n} J_{n}\left(\frac{2 \pi}{\lambda} r_{u}\right) e^{j n\left(\varphi_{u}-\varphi\right)} .
$$

By substituting (80) and (81) into (78), it can be obtained that

$$
Q_{k}=\frac{\lambda e^{-j \frac{2 \pi}{\lambda} r_{0}}}{4 \pi r_{0}} \sum_{u=1}^{U} \sum_{n=0}^{\infty} j^{n} J_{n}\left(\frac{2 \pi}{\lambda} r_{u}\right) \int_{0}^{2 \pi} e^{j n\left(\varphi_{u}-\theta\right)} e^{j k \theta} d \theta+\frac{o}{r_{0}}, \quad \text { a.s. }
$$

Based on the following equality

$$
\frac{1}{2 \pi} \int_{0}^{2 \pi} e^{j(k-n) \theta} d \theta=\delta_{k, n}
$$

the desired result follows from (82).

\section{B. Proof of Lemma 3}

Using trigonometric identities, it can be obtained that

$$
\begin{aligned}
r_{u} \cos \left(\varphi_{u}-\theta\right)-r_{k} \cos \left(\varphi_{k}-\theta\right) & =\left|X_{u}-X_{k}\right|\left(\cos \beta_{u, k} \cos \theta-\sin \beta_{u, k} \sin \theta\right) \\
& =\left|X_{u}-X_{k}\right| \cos \left(\beta_{u, k}+\theta\right)
\end{aligned}
$$

where the angle $\beta_{u, k}$ is defined by

$$
\tan \beta_{u, k}=\frac{r_{u} \cos \left(\varphi_{u}\right)-r_{k} \cos \left(\varphi_{k}\right)}{r_{u} \sin \left(\varphi_{u}\right)-r_{k} \sin \left(\varphi_{k}\right)}
$$

It follows that

$$
\begin{aligned}
\frac{1}{2 \pi} \int_{0}^{2 \pi} e^{j \frac{2 \pi}{\lambda}\left(r_{u} \cos \left(\varphi_{u}-\theta\right)-r_{k} \cos \left(\varphi_{k}-\theta\right)\right)} d \theta & =\frac{1}{2 \pi} \int_{0}^{2 \pi} e^{j \frac{2 \pi}{\lambda}\left|X_{u}-X_{k}\right| \cos \left(\beta_{u, k}+\theta\right)} d \theta \\
& =\frac{1}{2 \pi} \int_{0}^{2 \pi} e^{j \frac{2 \pi}{\lambda}\left|X_{u}-X_{k}\right| \sin \left(\beta_{u, k}+\theta+\frac{\pi}{2}\right)} d \theta .
\end{aligned}
$$

Using the definition of Bessel function in (68),

$$
\frac{1}{2 \pi} \int_{0}^{2 \pi} e^{j \frac{2 \pi}{\lambda}\left(r_{u} \cos \left(\varphi_{u}-\theta\right)-r_{k} \cos \left(\varphi_{k}-\theta\right)\right)} d \theta=j e^{j \beta_{u, k}} J_{m}\left(\frac{2 \pi}{\lambda}\left|X_{u}-X_{k}\right|\right) .
$$

Substituting (84) into (23) gives

$$
g\left(X_{k} \mid X_{u}\right)=\sqrt{\frac{P_{t}}{2 r_{0}}} j e^{j \beta_{u, k}} J_{0}\left(\frac{2 \pi}{\lambda}\left|X_{u}-X_{k}\right|\right)+\frac{o}{\sqrt{r_{0}}} .
$$

The desired result follows. 


\section{Proof of Lemma 5}

By substitution of the training signal in (4), its Laplace coefficients defined in (43) are obtained as

$$
\begin{aligned}
Q_{\ell}^{m} & =\frac{\eta}{r_{0}} \sum_{u=1}^{U} \int_{\theta=0}^{2 \pi} \int_{\psi=0}^{\pi} e^{j \frac{2 \pi}{\lambda} r_{u} \cos \psi_{u}} Y_{\ell}^{m}(\varphi, \theta) \sin \varphi d \varphi d \theta+ \\
& \int_{\theta=0}^{2 \pi} \int_{\psi=0}^{\pi} z(\varphi, \theta) \sin \varphi d \varphi d \theta+\frac{o}{r_{0}} \\
& =\frac{\eta}{r_{0}} \sum_{u=1}^{U} \int_{\theta=0}^{2 \pi} \int_{\psi=0}^{\pi} e^{j \frac{2 \pi}{\lambda} r_{u} \cos \psi_{u}} Y_{\ell}^{m}(\varphi, \theta) \sin \varphi d \varphi d \theta+\frac{o}{r_{0}}, \quad \text { a.s. }
\end{aligned}
$$

where the vanishment of noise follows similar analysis as in the proof for Lemma 1. Using the FunkHecke Theorem in (S2), the exponential term in the last equation can be also expanded into a Laplace series as

$$
e^{j \frac{2 \pi}{\lambda} r_{u} \cos \psi_{u}}=\sum_{\ell=0}^{\infty} \sum_{m=-\ell}^{\ell} c_{\ell} Y_{\ell}^{m}\left(\varphi_{u}, \theta_{u}\right) Y_{\ell}^{m}(\psi, \theta)
$$

where

$$
c_{\ell}=2 \pi \int_{-1}^{1} e^{j \frac{2 \pi}{\lambda} r_{u} \tau} P_{\ell}(\tau) d \tau
$$

Using Property (B6) in Appendix A, it follows from (87) that

$$
c_{\ell}\left(r_{u}\right)=\frac{(2 \pi)^{\frac{3}{2}} j^{\ell} J_{\ell+\frac{1}{2}}\left(\frac{2 \pi}{\lambda} r_{u}\right)}{\left(\frac{2 \pi}{\lambda} r_{u}\right)^{\frac{1}{2}}} Y_{\ell}^{m}\left(\varphi_{u}, \theta_{u}\right) .
$$

Combining (86) and (88) gives the desired result.

\section{Proof of Lemma 7}

Since $\psi_{u}$ is the angle between two unit vectors with spherical coordinates $(1, \varphi, \theta)$ and $\left(1, \varphi_{u}, \theta_{u}\right)$,

$$
\cos \psi_{u}=\sin \varphi \cos \theta \sin \varphi_{u} \cos \theta_{u}+\sin \varphi \sin \theta \sin \varphi_{u} \sin \theta_{u}+\cos \varphi \cos \varphi_{u}
$$

It follows that

$$
\begin{aligned}
r_{u} \cos \psi_{u}-r_{k} \cos \psi_{k}= & \sin \varphi \cos \theta\left(r_{u} \sin \varphi_{u} \cos \theta_{u}-r_{k} \sin \varphi_{k} \cos \theta_{k}\right)+ \\
& \sin \varphi \sin \theta\left(r_{u} \sin \varphi_{u} \sin \theta_{u}-r_{k} \sin \varphi_{k} \sin \theta_{k}\right)+ \\
& \cos \varphi\left(r_{u} \cos \varphi_{u}-r_{k} \cos \varphi_{k}\right) .
\end{aligned}
$$

To rewrite the right-hand side of (90) in a compact form, define the spherical coordinates $\left(r_{u, k}, \varphi_{u, k}, \theta_{u, k}\right) \in$ $\mathbb{R}^{3}$ such that

$$
\begin{aligned}
r_{u, k}^{2}= & \left(r_{u} \sin \varphi_{u} \cos \theta_{u}-r_{k} \sin \varphi_{k} \cos \theta_{k}\right)^{2}+\left(r_{u} \sin \varphi_{u} \sin \theta_{u}-r_{k} \sin \varphi_{k} \sin \theta_{k}\right)^{2}+ \\
& \left(r_{u} \cos \varphi_{u}-r_{k} \cos \varphi_{k}\right)^{2}
\end{aligned}
$$


and the angles $\varphi_{u, k}$ and $\theta_{u, k}$ as in the lemma statement. Moreover, let $\psi_{u, k}$ denote the angle between $\left(r_{0}, \varphi, \theta\right)$ and $\left(r_{u, k}, \varphi_{u, k}, \theta_{u, k}\right)$. Using these definitions, (90) can be reduced to

$$
r_{u} \cos \psi_{u}-r_{k} \cos \psi_{k}=r_{u, k} \cos \psi_{u, k}
$$

It can be obtained from (91) that $r_{u, k}^{2}=\left|X_{u}-X_{k}\right|^{2}$. Thus,

$$
r_{u} \cos \psi_{u}-r_{k} \cos \psi_{k}=\left|X_{u}-X_{k}\right| \cos \psi_{u, k} .
$$

Substituting this result into (53) yields

$$
\mathcal{J}_{u, k, m, \ell}=\iint e^{j \frac{2 \pi}{\lambda}\left|X_{u}-X_{k}\right| \cos \psi_{u, k}} Y_{\ell}^{m}(\varphi, \theta) \sin \varphi d \varphi d \theta
$$

Applying Lemma 5 gives the desired result.

\section{REFERENCES}

[1] China Mobile, "C-RAN: The road towards green RAN," White Paper, 2011.

[2] V. Chandrasekhar, J. G. Andrews, and A. Gatherer, "Femtocell networks: A survey," IEEE Comm. Magazine, vol. 46, no. 9, pp. 59-67, 2008.

[3] F. Rusek, D. Persson, B. K. Lau, E. G. Larsson, T. L. Marzetta, O. Edfors, and F. Tufvesson, "Scaling up mimo: Opportunities and challenges with very large arrays," IEEE Signal Proc. Magazine., vol. 30, no. 1, pp. 40-60, 2013.

[4] R. W. Heath Jr., S. Peters, Y. Wang, and J. Zhang, "A current perspective on distributed antenna systems for the downlink of cellular systems," IEEE Comm. Magazine, vol. 51, no. 4, pp. 161-167, 2013.

[5] W. Choi and J. G. Andrews, "Downlink performance and capacity of distributed antenna systems in a multicell environment," IEEE Trans. on Wireless Comm., vol. 6, no. 1, pp. 69-73, 2007.

[6] J. Zhang and J. G. Andrews, "Distributed antenna systems with randomness," IEEE Trans. on Wireless Comm., vol. 7, no. 9 , pp. 3636-3646, 2008.

[7] R. W. Heath, T. Wu, Y. H. Kwon, and A. C. K. Soong, "Multiuser mimo in distributed antenna systems with out-of-cell interference," IEEE Trans. on Signal Proc., vol. 59, no. 10, pp. 4885-4899, 2011.

[8] H. Zhu and J. Wang, "Radio resource allocation in multiuser distributed antenna systems," IEEE J. of Sel. Areas in Comm., vol. 31, no. 10, pp. 2058-2066, 2013.

[9] J. Zhang, C.-K. Wen, S. Jin, X. Gao, and K.-K. Wong, "On capacity of large-scale mimo multiple access channels with distributed sets of correlated antennas," IEEE J. of Sel. Areas in Comm., vol. 31, pp. 133-148, Feb. 2013.

[10] M. Matthaiou, C. Zhong, M. R. McKay, and T. Ratnarajah, "Sum rate analysis of zf receivers in distributed MIMO systems," IEEE J. of Sel. Areas in Comm., vol. 31, pp. 180-191, Feb. 2013.

[11] Z. Liu and L. Dai, "A comparative study of downlink mimo cellular networks with co-located and distributed base-station antennas," IEEE Trans. on Wireless Comm., vol. 13, no. 11, pp. 6259-6274, 2014.

[12] W. Feng, Y. Wang, N. Ge, J. Lu, and J. Zhang, "Virtual MIMO in multi-cell distributed antenna systems: coordinated transmissions with large-scale csit," IEEE J. of Sel. Areas in Comm., vol. 31, no. 10, pp. 2067-2081, 2013.

[13] I. E. Telatar, "Capacity of multi-antenna gaussian channels," European Trans. on Telecomm., vol. 10, no. 6, pp. 585-595, 1999.

[14] P. Z. Wang, Xinzheng and M. Chen, "Antenna location design for generalized distributed antenna systems," IEEE Comm. Letters, vol. 13, pp. 315-317, May 2009.

[15] E. Park, S.-R. Lee, and I. Lee, "Antenna placement optimization for distributed antenna systems," IEEE Trans. on Wireless Comm., vol. 11, pp. 2468-2477, Nov. 2012.

[16] A. S. Y. Poon, R. W. Brodersen, and D. N. C. Tse, "Degrees of freedom in multiple-antenna channels: A signal space approach," IEEE Trans. on Information Theory, vol. 51, no. 2, pp. 523-536, 2005. 
[17] F. K. Gruber and E. A. M., "New aspects of electromagnetic information theory for wireless and antenna systems," IEEE Transactions on Antennas and Propagation, vol. 56, pp. 3470-3484, Nov. 2008.

[18] T. L. Marzetta, "Noncooperative cellular wireless with unlimited numbers of base station antennas," IEEE Trans. on Wireless Comm., vol. 9, no. 11, pp. 3590-3600, 2010.

[19] K. M. Buckley and B. D. Van Veen, "Beamforming: A versatile approach to spatial filtering," IEEE ASSP Magazine, vol. 5, no. 2, pp. 4-24, 1988.

[20] T. S. Rappaport, Wireless Communications: Principles and Practice. Prentice Hall, 2001.

[21] A. Yang, Y. Jing, C. Xing, Z. Fei, and J. Kuang, "Performance analysis and location optimization for massive mimo systems with circularly distributed antennas," Available: http://arxiv.org/abs/1408.1468.

[22] H. L. Van Trees, Detection, estimation, and modulation theory. John Wiley \& Sons, 2004.

[23] H. Yin, D. Gesbert, M. Filippou, and Y. Liu, "A coordinated approach to channel estimation in large-scale multiple-antenna systems," IEEE J. of Sel. Areas in Comm., vol. 31, pp. 264-273, Feb. 2013.

[24] H. T. Friis, “A note on a simple transmission formula," Proc. of IRE, vol. 34, pp. 254-256, May 1946.

[25] G. B. Arfken, H. J. Weber, and F. E. Harris, Mathematical Methods For Physicists: A Comprehensive Guide. Academic Press, 7 ed., 2012.

[26] L. C. Andrews, Special functions of mathematics for engineers. McGraw-Hill, Inc., 2 ed., 1992.

[27] L. Landau, "Bessel functions: monotonicity and bounds," Journal of the London Mathematical Society, vol. 61, no. 1, pp. 197-215, 2000.

[28] M. Abramowitz and I. A. Stegun, Handbook of Mathematical Functions with Formulas, Graphs, and Mathematical Tables. New York: Dover: Dover Publications, 1965.

[29] M. Abramowitz and I. A. Stegun, Handbook of mathematical functions: with formulas, graphs, and mathematical tables. Courier Dover Publications, 1972.

[30] N. A. Gumerov and R. Duraiswami, Fast multipole methods for the Helmholtz equation in three dimensions. Elsevier, 2005.

[31] T. Strohmer and R. W. Heath Jr, "Grassmannian frames with applications to coding and communication," Applied and computational harmonic analysis, vol. 14, no. 3, pp. 257-275, 2003.

[32] D. Stoyan, W. S. Kendall, and J. Mecke, Stochastic Gemoetry and its Applications. Wiley, 2nd ed., 1995. 